Discussion papers of the

Max Planck Institute for

Research on Collective Goods

Bonn 2019/8

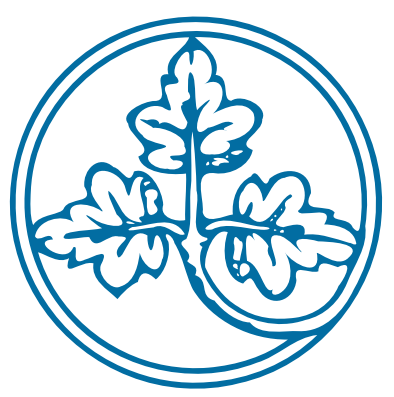

An Experimental Study on the Effects of Communication, Credibility, and Clustering in Network Gamess

Gary Charness

Francesco Feri

Miguel A. Meléndez-Jiménez

Matthias Sutter

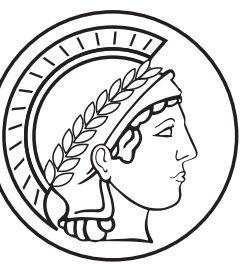




\section{An Experimental Study on the Effects of Communi- cation, Credibility, and Clustering in Network Games}

Gary Charness / Francesco Feri / Miguel A. Meléndez-Jiménez / Matthias Sutter

May 2019 


\title{
An Experimental Study on the Effects of Communication, Credibility, and Clustering in Network Games*
}

\author{
Gary Charness, Francesco Feri, Miguel A. Meléndez-Jiménez, \\ and Matthias Sutter\#
}

May 12, 2019

\begin{abstract}
The effectiveness of social interaction depends strongly on an ability to coordinate actions efficiently. In large networks, such coordination may be very difficult to achieve and may depend on the communication technology and the network structure. We examine how pre-play communication and clustering within networks affect coordination in a challenging experimental game on eight-person networks. Free-form chat is enormously effective in achieving the nonequilibrium efficient outcome in our game, but restricted communication (where subjects can only indicate their intended action) is almost entirely ineffective. We can rationalize this result with a novel model about the credibility of cheap-talk messages. This credibility is much larger with freeform message communication than with restricted communication. We are the first to model this credibility and show, both theoretically and experimentally, an interaction effect of network structure and communication technologies. We also provide a model of message diffusion, which indeed predicts that diffusion will be more rapid without clustering and is consistent with our data.
\end{abstract}

JEL Codes: C71, C91, D03, D85

Keywords: Networks, Clustering, Communication, Credibility, Cheap talk, Experiment

\footnotetext{
* We thank Ramon Cobo-Reyes, Brit Grosskopf, Armin Falk, Sanjeev Goyal, Erik Kimbrough, Arthur Robson, seminar participants at the 2016 ASSA meetings, University of Bonn (2016), CESifo, UCSB, Simon Fraser University, Stanford Institute for Theoretical Economics, the University of Cologne, Columbia University, University of East Anglia, University of Exeter, LBEG at LSE, the EEG-inaugural conference in Bonn (2018), the III BiNoMa workshop on economics of networks in Bilbao and the University of Portsmouth for helpful comments. Funding from Ministerio de Ciencia, Innovación y Universidades, Spain (ref. RTI2018-097620-B-I00) and the University of Cologne is gratefully acknowledged.

\# Charness, University of California at Santa Barbara, IZA Bonn, and CESifo Munich, charness@econ.ucsb.edu. Feri, Royal Holloway University of London and University of Trieste, Francesco.Feri@rhul.ac.uk. Meléndez-Jiménez, Universidad de Málaga, melendez@uma.es. Sutter, Max Planck Institute, Bonn, University of Cologne and University of Innsbruck, matthias.sutter@coll.mpg.de.
} 


\section{Introduction}

In many field environments, agents must coordinate their actions to achieve success. For example, collective action may only be effective when a threshold number of parties choose to participate in an action that is potentially risky. Think of hunting together or defending one's territory against potential invaders, coordinating on a ceasefire in military conflict, or imagine companies coordinating on a new technological standard when competing platforms are available (AlosFerrer et al., 2010). Naturally, communication amongst the parties who are potentially involved may occur; yet this communication may need to be covert and so may be restricted to those people with whom one is directly linked. This creates a network setting in which individuals can interact only with other individuals who share a connection or link. Such networks - which form a bridge between bi-lateral interactions and full-fledged markets in which all agents can interact with all other agents - are ubiquitous and important for many domains in one's life. Accordingly, Jackson (2010) states (p. 512) that one's social network "influences patterns of decisions regarding education, career, hobbies, criminal activity, and even participation in micro-finance.”

There is even evidence that social-media networks (e.g., Facebook and Twitter) have had a crucial role in the effectiveness of revolutions (e.g., the Arab Spring or the Hong Kong protests; see Parker, 2014). People might wish to participate in revolutions if enough others also participate, in which case the information flow in one's network may become decisive. ${ }^{1}$ There could be one risky action that denotes active participation, a safe action of not participating, and perhaps some intermediate action like giving support to the revolution without actively participating. Another example for a collective-action problem with private prior communication is the decision by a firm's workers to go on strike. Consider for example a situation in which each worker talks to his colleagues in the firm the day before the possible strike in order to make up his mind whether or not to support the strike the next day.

These examples are complicated real-life situations with potentially many equilibria - so it is difficult to predict what will happen and it seems plausible that the network structure and the scope of communication among potentially-involved parties crucially impact the likelihood of successfully coordinating their actions. Given that the real-world examples discussed above are

\footnotetext{
${ }^{1}$ For example, with Facebook one can see one's friends' messages and vice versa. People can resend messages (or give likes), and this information can then diffuse through the social network, triggering different kinds of actions.
} 
too complex for one to carefully control the network structure and the communication between parties, we study such situations in detail in an abstract and controlled laboratory setting. Such a setting allows us to examine how network characteristics such as clustering and how different forms of pre-play communication - and the interaction between network structure and communication forms - affect coordination in a challenging network game.

In our experiment, we form 8-person networks in which each person is linked to exactly four others. Only players who are linked can communicate with each other (see below for the forms of communication) and then play an extended stag hunt game in which we select (at random) one of the four neighbors as the interaction partner (strategically equivalent to a playing-the-field game). The extended stag hunt creates a challenging and novel game. It builds on the classic stag hunt game (Rousseau, 1755, 1988; Aumann, 1990; Charness, 2000) with two actions per player and two Pareto-ranked equilibria. In our extension, we add a third action that is strictly dominated by both other actions and so preserves the pure-strategy equilibria of the classic game. When played jointly, this third action leads to the highest social payoffs, but can never be a best response (in own payoffs) and one receives a zero payoff by choosing it when the other player does not. ${ }^{2}$

Our design varies two factors and the unique combination separates our paper from previous work and sheds light on the underpinnings of behavior and efficient play in networks. First, we allow for communication (Crawford, 1998) and vary the communication technology by permitting (i) no communication, (ii) simple letter messages (closed), or (iii) free-form chat messages (open) with the persons to whom one is linked. Second, we vary the degree of clustering by implementing networks with (i) zero clustering, and (ii) positive clustering. Clustering is considered critical in terms of network structure, measuring the extent to which an individual's neighbors in a network are linked. In one of our networks - the clustered network - each of a subject's four neighbors is linked at least to another one of his neighbors. In the other - nonclustered - network none of a subject's neighbors is linked to another one of his neighbors.

Concerning the first factor, communication, it is noteworthy that, in our extended stag hunt game, none of these technologies should be sufficient to achieve the socially-efficient (but dominated) outcome, since standard theory doesn't consider that messages will be credible when

\footnotetext{
${ }^{2}$ Our extension has some similarity to what Cooper et al. (1992; see their Figure I) call the cooperative coordination game, where the third action - if played mutually - also yields the highest social payoffs. Yet, in their case the third action is not strictly dominated by both other actions. Hence, our extension makes it more challenging to reach the social optimum.
} 
interests are sufficiently opposed (the third action is dominated by both others). However, we present two behavioral models that address how communication might affect play in the network game, and we then derive predictions from these models for our experiment. For both models, we assume that there is a taste for efficiency (Charness and Rabin, 2002). We start with a model of the diffusion of messages within the network, which will yield predictions about the effects of our treatment variation with respect to the clustering coefficient of the two networks. We then proceed with a model of the credibility of messages that will help us to form predictions about different effects of open and closed communication. The latter is a novel feature, demonstrating why open and closed communication could yield different effects on actual play.

Previous work has almost completely ignored the effects of communication in networks. To the best of our knowledge, at this writing there is only one experimental paper that considers the issue of communication in networks. Choi and Lee (2014) studies coordination in networks in a four-player version of the battle of the sexes, investigating the trade-off between the efficiency and equity of coordination outcomes and its link to the network structure of communication in four types of networks (complete, star, kite, and line). The four players engage in multiple periods of closed cheap talk among connected members about their intended actions in the underlying game (with four possible signals available, one for each of the positions). They find that their closed communication tends to induce coordination on the equilibrium preferred by the best-connected players. Still, we are the first to introduce rich (open) communication to a network environment, and we also consider a second factor by varying the degree of clustering in the network, while keeping all other elements of the network structure identical. ${ }^{3}$

Previous work has examined the effects of clustering on play in network games. Models by Eshel et al. (1998), Assenza et al. (2008), Vega-Redondo et al. (2005), and Vega-Redondo (2006) all predict that a higher degree of clustering will lead to better cooperation. Experimental work by Berninghaus et al. (2002), Keser et al. (1998), Cassar (2007), Charness et al. (2014), and Melamed et al. (2018) all find that higher clustering leads to more efficient outcomes. Yet, none of these experiments has network-member communication, and we are not aware of any paper that has considered how clustering in networks interacts with different forms of communication in

\footnotetext{
${ }^{3}$ Choi and Lee (2014) change both clustering and connectivity (which is the extent to which the nodes of a network are connected) at the same time which makes it impossible to study the effects of clustering on the diffusion of messages in the communication stage and on actual play. As we will argue, the interaction of clustering and communication offers important insights into how groups of people can achieve efficient outcomes.
} 
terms of the rate of cooperative play induced. We are particularly interested in how messages to play cooperatively diffuse across the two different networks. For this purpose, we build on an idea of Kearns et al. (2009) who let participants in an experimental network game have a period of time (60 seconds) to indicate their intended action. During this period, they see in real time the other members' messages and can change their own expressed intention at any time. Yet, the last message before the period ends is the action actually played in the game of Kearns et al. (2009), which implies that not all members can react to other members' last messages and that communication is not cheap talk, but is actually implemented. In our design, we let subjects exchange (and change) messages for 90 seconds; they then make simultaneous choices in the game. This extended period allows us to examine how messages diffuse through the network and how this affects actual play, contingent on the network structure.

Regarding this network structure, changing the degree of clustering in the network could in principle have two opposing effects on the diffusion of messages. When the clustering among neighbors is high, agents observe their neighbors responding to similar conditions and play as if they were playing in a small group instead of a much larger network. This follows the previous suggestion of Holland and Leinhardt (1971) and Watts and Strogatz (1998) that nodes in social networks tend to create tightly-knit groups. High clustering may then protect cooperative players from potential defectors that are likely to be around in larger networks. This may yield higher cooperation in networks with higher clustering. However, there is also an opposite effect since networks with lower (or even no) clustering may promote a quicker diffusion of communication content in the network since one member's message reaches (in our case four) other members who have yet other new neighbors so that messages can spread faster through the whole network.

In fact, we develop and present a theoretical model that shows that the diffusion of messages within the network is slower with positive clustering and thus affects the distribution of messages before subjects make decisions. Our two experimental networks support the model's predictions; we also consider how the diffusion of communication affects actual play in the networks. At the time of writing, we are the first to study the interaction of the network structure with the diffusion of information from communication.

In addition to studying the diffusion of messages across networks, it is at least equally important to examine how credible such messages are, and doing so is another novelty of our paper. While theoretical papers have discussed issues such as neologism-proofness (Farrell, 1993; 
Matthews et al., 1991), ${ }^{4}$ self-committing (Farrell and Rabin, 1996), ${ }^{5}$ self-enforcing (Aumann, 1990), or credible message rationalizability (Rabin, 1990), ${ }^{6}$ there is little experimental work investigating the critical issue of what makes a message credible. ${ }^{7}$ We present a model about the credibility of messages that predicts free-form messages to be more credible than restricted forms of communication (like signaling one's intended action). Then we test this prediction by constructing a credibility indicator that attempts to measure credibility in an objective way as a function of a subject's past experiences and behavior.

Our results regarding communication are quite striking. In our extended stag hunt game, we find attempts at coordinating on the socially-efficient-but-risky non-equilibrium outcome in the early rounds of each treatment, i.e., without communication, with closed communication (only indicating the intended strategy), and with open, free-form chat communication. Yet, the rate of cooperative play to achieve the socially-efficient outcome rapidly deteriorates to almost nothing without communication and does so just a bit more slowly with closed communication than with no communication. On the other hand, the rate at which people achieve the socially-efficient outcome with open communication starts high and continues to be well over $90 \%$ in these sessions, compared to less than $20 \%$ in the other treatments.

The success of open communication is higher in the network with zero clustering, perhaps reflecting the quicker diffusion of messages that strive for the socially-efficient outcome. This result supports the theoretical predictions of our model and we consider it an important result, since it shows an interaction of network structure and communication technology. Comparing open communication to closed communication, we also find that messages in open communication have a significantly higher credibility indicator than messages in the closed communication treatment, which is in line with our theoretical predictions. In fact, while open communication has been shown to increase cooperation in bilateral interactions before (e.g., Brosig et al., 2003; Charness

\footnotetext{
${ }^{4}$ Farrell (1993) defines neologisms to be “messages that were not expected in equilibrium” (p. 515) and neologismproofness to be a condition in which "no such credible neologism is available and attractive relative to the equilibrium" (p. 515). But credible neologisms are in fact present in our messages and in other message data.

5 Farrell and Rabin (1996) define a message to be self-committing if it creates incentives for the signaler to fulfill it, if the other player considers the message credible.

${ }^{6}$ Paraphrasing Crawford (1998), this is a non-equilibrium concept featuring a neologism-proof credibility restriction and an assumption that players maximize their expected payoffs given their beliefs.

${ }^{7}$ Exceptions include Blume et al. (1998), who consider neologism-proofness and find that it is difficult to keep subjects from smuggling a common language into the experiment; Charness and Dufwenberg (2006), who find that promises tend to be believed and honored; and Brandts et al. (2016), who find that establishing a friendly rapport and making specific promises are quite helpful. Yet none of these studies really shed that much light on credibility.
} 
and Dufwenberg, 2006, 2011; Ben-Ner et al., 2011; Brandts et al., 2016), ours is the first paper to demonstrate such a strong effect in a complicated network structure and challenging game (our extended stag hunt game).

Closed communication is almost completely ineffective in the network case, which stands in stark contrast to earlier work in simpler, non-network settings. Closed communication in the form of sending messages about intended actions has been frequently found to increase efficiency in coordination games (e.g., Cooper et al., 1992; Charness, 2000; Clark et al., 2001; Blume and Ortmann, 2007). The failure of closed communication to increase cooperation and efficiency in our setting may be due to the complex network and communication structure. As evidence for this point we also present a robustness check with another treatment in which we let subjects play our extended stag hunt game with bilateral matching. In line with the previous findings about closed communication in bilateral settings, closed communication, in our treatment with bilateral matching, is also very effective in raising the level of cooperative play above the level prevailing without communication.

We observe only small-to-modest differences in play across our network-clustering conditions with either no communication or closed communication. While clustering seems to help cooperation with no communication, in line with previous work discussed above, this reverses with open communication. The differences are modest, but statistically-significant in our regressions. This result with open communication suggests that the opposite effects of clustering regarding play and communication are countervailing, with the negative effect of positive clustering (due to slower diffusion of messages) prevailing overall.

Our main takeaways from our results are: 1) open communication is tremendously successful in achieving the socially-efficient and non-equilibrium outcome even in a relatively difficult network environment, 2) closed communication does not achieve high levels of cooperation, although it is fairly effective in the standard Stag Hunt, 3) we find evidence of diffusion of messages to be contingent on the degree of network clustering, 4) the credibility of messages depends on the type of communication, and 5) comparing across networks, we find evidence of a negative effect of clustering on efficiency when communication is open.

The reminder of the article is organized as follows. We describe the experimental design and implementation in Section 2. Section 3 presents two models on diffusion and credibility of 
messages in networks and our hypotheses for the experiment. The experimental results are described in detail in Section 4, and Section 5 concludes.

\section{Experimental design and implementation}

\subsection{Experimental design}

Subjects are arranged on an 8-node symmetric network to play a game with one of their neighbors. Each subject in the network is linked to four neighbors and communication is only possible with one's neighbors. Each subject plays the game shown in Figure 1 with one randomlyselected neighbor. Figure 1 represents an extended Stag Hunt game, where strategy profile $(A, A)$ represents the risk-dominant equilibrium, $(B, B)$ represents the payoff-dominant equilibrium and (C,C) represents the socially-efficient outcome. This is clearly not an equilibrium, since strategy C is strictly (weakly) dominated by strategy A (B).

Figure 1: The extended Stag Hunt game (ABC)

\begin{tabular}{ccccc}
\hline \hline & & \multicolumn{3}{c}{ Player 2 } \\
& & A & B & C \\
\cline { 3 - 5 } Player 1 & B & 70,70 & 80,0 & 110,0 \\
& C & 0,80 & 90,90 & 110,0 \\
& & 0,110 & 0,110 & 100,100 \\
\hline \hline
\end{tabular}

Subjects play this game for 40 periods, and the timing is as follows in each period:

1. At the beginning of each period, subjects are randomly assigned to a group of 8 and to one node of the network. Each node has links to four neighbors.

2. Prior to playing the game, one can communicate (when communication is allowed) with one’s neighbors for 90 seconds. Within this period, players can send a message (or in the case of closed communication indicate the intended action) at any point and neighbors continuously observe the signals of all neighbors. Within the 90 seconds, messages can 
also be changed. No more messages can be sent when the period ends, and the final message stands.

3. Each subject chooses one action (either A, B, or C).

4. Subjects are randomly paired with one of their neighbors. For each pair the chosen actions define the individual payoffs. Players are informed about the action of the paired player and the monetary outcome.

The two following treatment variations are implemented in a between-subjects design.

Treatment variation 1: Degree of clustering in the network.

Players are arranged in one of the two networks shown in Figure 2 and they are told their position in the network and the network structure. The two networks differ with respect to clustering, one of the features considered important in terms of network structure, and all other elements are kept the same. This means that both networks are identical regarding connectivity, diameter, and assortativity. ${ }^{8}$ A cluster refers to a triple of subjects that satisfy the condition that each person in the cluster is connected to the other two members of the cluster.

The two networks in Figure 2 are characterized as follows:

- Clust: In the network on the left-hand side of Figure 2, each one of a subject's neighbors is linked at least to another one of his neighbors. This network has a positive cluster coefficient of 0.5 . The clustering coefficient $\mathrm{C}$ is defined as the ratio of the number of closed triplets to the number of all triplets (open and closed).

- NoClust: Here, on the right-hand side of Figure 2, none of a subject's neighbors is linked to another one of his neighbors. This network has therefore zero clustering (i.e., a cluster coefficient of zero).

\footnotetext{
${ }^{8}$ Both networks have eight players and they are homogenous of degree 4 (all players have four links), so they have the same connectivity and assortativity. Furthermore, both networks are symmetric (immune to permutations) and have diameter 2 (any two players can reach each other by a path not greater than two links).
} 
Figure 2: The networks

Network Clust (High Clustering) Network NoClust (No Clustering)

Treatment variation 2: Communication within the network

We also vary the form of communication that is permitted in a session. We have three communication protocols:

- No communication: Subjects play the game without any form of communication between them.

- Closed communication (Simple letter messages): Participants send messages by marking an option A, B or C. Messages are immediately visible to all her neighbors and each participant can change the marked option at any time during the period of 90 seconds that precede the game.

- Open communication (Free-form chat messages): One sends a message via chat that is immediately visible to all neighbors. Again, one can send as many messages as desired during this stage (of 90 seconds), and can also revoke or change messages.

Our design is one of local payoffs with local communication. An agent's payoffs depend on the decisions made by his neighbors, since ex-post (once the actions are already chosen) each player is randomly paired with one of his neighbors, and the payoffs in a pair are determined by the actions chosen by the two players. Note also that this "random pairing" is strategically equivalent to a playing-the-field game (where one plays the same action in the game with all neighbors and one's total payoff is the average of one's payoffs in each bilateral interaction). The random pairing, however, constitutes the most challenging environment and makes coordination 
on C very difficult, as participants face the additional uncertainty regarding with which of their four neighbors they will actually play. In a playing-the-field game, this uncertainty is removed and may make it easier to play action C. Hence, in some sense our design is deliberately biased against finding high rates of C-play.

\subsection{Implementation}

Table 1 shows information about the sessions we conducted. There were 32 people in each session (except for one session in treatment Closed_Clust), each with two separate matching groups of 16 , so the number of purely-independent observations for each treatment is thus the number of participants divided by 16 . In each period the 16 participants of a matching group were randomly assigned to one of two 8-person groups. Moreover, in each 8-person group positions in the network were randomly distributed. No one knew the identity of any other player. A sample of our instructions (translated into English) is shown in Appendix A.

\section{Table 1: Treatments, sessions and participants}

\begin{tabular}{llcc}
\hline \hline Description of treatment & Treatment abbreviation & Sessions & Participants \\
No communication, clustered network & NoComm_Clust & 2 & 64 \\
No communication, non-clustered network & NoComm_NoClust & 2 & 64 \\
Closed communication, clustered network & Closed_Clust & 2 & 48 \\
Closed communication, non-clustered network & Closed_NoClust & 2 & 64 \\
Open communication, clustered network & Open_Clust & 2 & 64 \\
Open communication, non-clustered network & Open_NoClust & 2 & 64 \\
Closed communication, bilateral & Closed_Bilateral & 2 & 64 \\
\hline \hline
\end{tabular}

Since one is linked to four players in terms of messages, but only is matched with one of them in the game, our network environment introduces considerable uncertainty regarding the behavior of the to-be-matched player; furthermore, this uncertainty is compounded by the unseen 
messages received by one's neighbors (although there is diffusion of messages). As a robustness check and in order to remove this uncertainty, we ran treatment Closed_Bilateral, where we have closed communication involving bi-lateral matching rather than the four-link network format. We kept other features of the network environment the same to facilitate a clean comparison. We suspected that the uncertainty characterizing the network environment is an obstacle to efficient coordination of actions and therefore ran treatment Closed_Bilateral as a control. Yet, given our results with open communication in the network environment (see below), we only ran the bilateral matching treatment with closed communication.

In total, we had 432 people in our sessions that were run at the University of Cologne. The average duration was approximately 80 minutes without communication and 120 minutes with communication. The average amount earned was 19.1 Euro and 22.8 Euro, respectively, including a show-up payment of 4 Euro.

\section{Theoretical models and experimental hypotheses}

In this section, we consider two models that shed light on the effect of communication on play in the network game. For both models, we assume that there is a taste for efficiency: players like to get the efficient outcome $(C, C)$. We begin with a model of the diffusion of messages within the network, which gives predictions about the effects of changing the clustering coefficient across the two networks. We then proceed with a model of the credibility of messages that leads to predictions about play with open and closed communication.

\subsection{A model of diffusion of messages}

Assume that the communication stage is divided into a finite number of intervals $t \in$ $\{1,2, \ldots T\}$. In $t=1$ one player, denoted the seed player, sends a C-message (which is motivated by a possible increase in own payoffs and by a desire for efficiency; see Charness and Rabin, 2002). Her four neighbors receive the seed player's message. Then, in each interval $t \geq 2$ each player sends a $C$ message with probability $p \in(0,1)$ if she received at least one $C$ message in a previous interval. We assume that messages sent in interval $t$ are received in interval $t+1$. 
Proposition 1. In each interval $t \geq 3$ of the chat period, for each $p \in(0,1)$ the probability distribution of the number of subjects that have sent a $C$-message up to interval $t$ (included) in network NoClust first-order stochastically dominates (FOSD) the distribution of the number of subjects that have sent a C-message in network Clust.

The proof is in Appendix B. The main factor behind the proposition is that, given that both networks have the same connectivity, C-messages diffuse faster in the network without clusters (i.e., in NoClust) than when the clustering coefficient is positive (i.e., in Clust). Note that the value of probability $p \in(0,1)$ may depend on the type of communication. We could assume that this probability is larger in open communication than in closed communication. The justification is that in open communication the senders have more opportunities to convince the receivers, because they are not confined to signaling the intended action only. Yet, irrespective of whether the probability $p$ depends on the type of communication, our Proposition 1 leads to our first hypothesis.

Hypothesis 1. At the end of the chat period the observed distribution on the number of subjects that sent a C-message in network NoClust first-order stochastically dominates (FOSD) the distribution in network Clust.

The distribution of messages itself, however, may not affect actual play. Rather, actual play may depend on the credibility of transmitted messages, because as long as messages remain pure cheap talk, one would not expect any difference in actual play between the different networks and between treatments without communication and treatments with communication.

\subsection{A model of the credibility of messages}

This model is based on forward induction reasoning. In addition to the assumption of a taste for efficiency, which will be represented by a utility bonus $b>0$ that players get in case they achieve the efficient outcome (C,C), the model needs two further assumptions.

First, we assume that players incur a cost of lying (Gneezy et al., 2018; Abeler et al., 2019) if they play something different from what they announced in their message. This cost is positive both in open and in closed communication, but we assume it to be higher in open communication, in which messages are not pre-specified but directly produced by subjects. There is evidence in economics that the act of choosing serves as a form of commitment that enhances performance. This endogenous choice is a key factor in Sutter et al. (2010) and Dal Bó et al. (2010). The cleanest 
example of this is presented in Babcock et al. (2015), where people are given a choice between two environments. Since one of these essentially dominates the other, $97 \%$ of the subjects chose one environment. Their performance in this environment is $20 \%$ better than their performance when they had simply been assigned to this treatment. The media-richness literature in psychology (e.g., Dennis et al., 1999; Rockmann and Northcraft, 2018) also suggests that people are more likely to honor a claim or promise when this has been self-formulated. The cost of lying for open communication is $x^{\text {open }}>0$ and for closed communication it is $x^{\text {closed }}>0$, with $x^{\text {open }}>x^{\text {closed }}$.

Second, for simplicity and ease of illustration, we consider a two-player game in which one player (player 1) can send one message (M) or not send any message (NoM), and then, the Extended Stag Hunt game presented in Figure 1 is played. We will assume that the message represents the intention to play the efficient action (i.e., the message is "to play C" in our game).

Proposition 2. For each communication protocol $i \in\{$ open, closed $\}$, and $b>10$, there exists a threshold $\bar{x}$ such that, applying a forward induction argument, the message to play efficiently is credible if and only if $x^{i}>\bar{x}$.

There exists a set of parameters such that, by applying forward-induction arguments, open communication leads to credible messages on efficient play, while closed communication does not yield credible messages.

The proof is in Appendix B. ${ }^{9}$ Our Proposition 2 leads to our second hypothesis:

Hypothesis 2. Open communication will significantly increase the rate of cooperation on $C$, and more so than closed communication. Since closed communication may not be credible, it needs not yield higher rates of C-play than no communication.

Moreover, given the higher credibility of C-messages in open communication and the faster diffusion of such messages (see Hypothesis 1) we can state our third hypothesis:

Hypothesis 3. There will be a higher rate of C-play in NoClust than in Clust in case of open communication. Given previous results without communication, this is reversed without communication and possibly with closed communication. ${ }^{10}$

\footnotetext{
${ }^{9}$ The forward-induction arguments, introduced by Kohlberg and Mertens (1986), are based on the deletion of (weakly) dominated strategies, and were applied to the case where players can send costly signals prior to playing the game by, e.g., van Damme (1989) and Ben-Porath and Dekel (1992), and later studied in the lab by Huck and Müller (2005). Note that, in the presence of lying costs, messages can be viewed as costly signals.

${ }^{10}$ Recall that treatment Closed_Bilateral is a robustness check to see whether bilateral matching - rather than play in networks - makes a difference by removing the uncertainty concerning with whom one is matched. For this treatment with bi-lateral matching we expect that C-play will be more frequent than in the network treatments.
} 


\section{Experimental results}

\subsection{Descriptive statistics of actions}

Table 2 shows summary statistics of chosen actions over all 40 periods. ${ }^{11}$ Subjects are overwhelmingly likely to make the safe choice of A when communication is not feasible, and this is largely unchanged with closed communication. The rates of choosing action A range from $80.6 \%$ to $89.1 \%$ across the first four columns, which show data for no communication and closed communication, separately for both networks. We see very little B-play, even though $(B, B)$ is an equilibrium in standard theory. It is played in less than $5 \%$ of cases in the first four columns. ${ }^{12}$

Table 2: Likelihood of actions A, B and C, by treatment

\begin{tabular}{ccccccc}
\hline \hline & \multicolumn{2}{c}{ No Communication } & \multicolumn{2}{c}{ Closed Communication } & \multicolumn{2}{c}{ Open Communication } \\
& Clust & NoClust & Clust & NoClust & Clust & NoClust \\
\cline { 2 - 7 } A & $89.10 \%$ & $86.13 \%$ & $84.64 \%$ & $80.55 \%$ & $6.68 \%$ & $0.86 \%$ \\
& $(2281)$ & $(2205)$ & $(1625)$ & $(2062)$ & $(171)$ & $(22)$ \\
B & $2.23 \%$ & $4.41 \%$ & $2.19 \%$ & $4.26 \%$ & $0.82 \%$ & $0.08 \%$ \\
& $(57)$ & $(113)$ & $(42)$ & $(109)$ & $(21)$ & $(2)$ \\
C & $8.67 \%$ & $9.45 \%$ & $13.18 \%$ & $15.20 \%$ & $92.50 \%$ & $99.06 \%$ \\
& $(222)$ & $(242)$ & $(253)$ & $(389)$ & $(2368)$ & $(2536)$ \\
Total & $100 \%$ & $100 \%$ & $100 \%$ & $100 \%$ & $100 \%$ & $100 \%$ \\
& $(2560)$ & $(2560)$ & $(1920)$ & $(2560)$ & $(2560)$ & $(2560)$ \\
\hline \hline
\end{tabular}

The bottom number in each cell (in parentheses) is the number of observations.

\footnotetext{
${ }^{11}$ Table C1 in Appendix C breaks down the aggregate data into blocks of ten periods each.

12 The rarity of B-play in closed communication might seem surprising at first sight, given previous results that closed communication (indicating intended choices) increases the frequency of choosing a payoff-dominant equilibrium over the risk-dominant one in coordination games (e.g., Charness, 2000; Blume and Ortmann, 2007). The rarity is the consequence of extending the traditional stag-hunt game with a third, dominated, option. To show this, we ran additional treatments with the traditional stag-hunt game where subjects can only choose between option A and option $\mathrm{B}$ (the payoff table is the one from our Figure 1 if the row and column for action $\mathrm{C}$ are deleted). While without communication, option A is chosen in almost $90 \%$ of cases, closed communication raises the frequency of B-play to $75 \%$ of cases. Thus, our additional control treatments replicate former results, and the rarity of B-play in the extended stag-hunt game of Figure 1 is not an artifact of our subject pool, but due to the much more demanding game. Section 4.5 presents more details on our control treatments of the traditional stag-hunt game.
} 
The results with open communication are dramatically different. Even in this complex game played on a network, the cooperative play of $\mathrm{C}$ is remarkably frequent at $92.5 \%$ in the clustered network and at even $99.1 \%$ in the non-clustered network.

Conservative non-parametric tests (using each 16-person matching group as one observation) show clear statistical significance in the difference in the rate of cooperative C-play between open communication and each of the other communication protocols. Each of the opencommunication matching-groups had a higher $\mathrm{C}$ rate than any of the other groups. A one-tailed ranksum test indicates the differences between closed and open communication are significant for each network ( $p=0.017$ in network Clust, $p=0.010$ in network NoClust). Likewise, the difference between open and no communication are significant in each network ( $p=0.021$ for NoComm_Clust versus Open_Clust and $p=0.020$ for NoComm_NoClust versus Open_NoClust). However, there is no significant difference in C-play between closed and no communication ( $p=0.289$ for NoComm_Clust versus Closed_Clust and $p=0.248$ for NoComm_NoClust versus Closed_NoClust). These findings provide strong support for our Hypothesis 2, according to which C-play should be most frequent with open communication, and significantly lower otherwise.

One might imagine that people are initially optimistic about reaching the $(\mathrm{C}, \mathrm{C})$ outcome, but that this is soon tempered by receiving no payoffs after mis-coordinating. In fact, we see a time trend consistent with this in Figure 3. ${ }^{13}$ We see many choices of C (about half) initially in both the no-communication and closed-communication treatments, but this drops quickly in both cases (more so without communication). On the contrary, C is played almost $100 \%$ of the time with open communication, apart from slightly lower frequencies in the first period and the last few periods (in which unraveling is not surprising, since this is not a pure coordination game).

\footnotetext{
${ }^{13}$ Table C2 and Figure C1 in Appendix C breaks this out by matching groups.
} 
Figure 3: Frequencies of actions across periods, by treatment

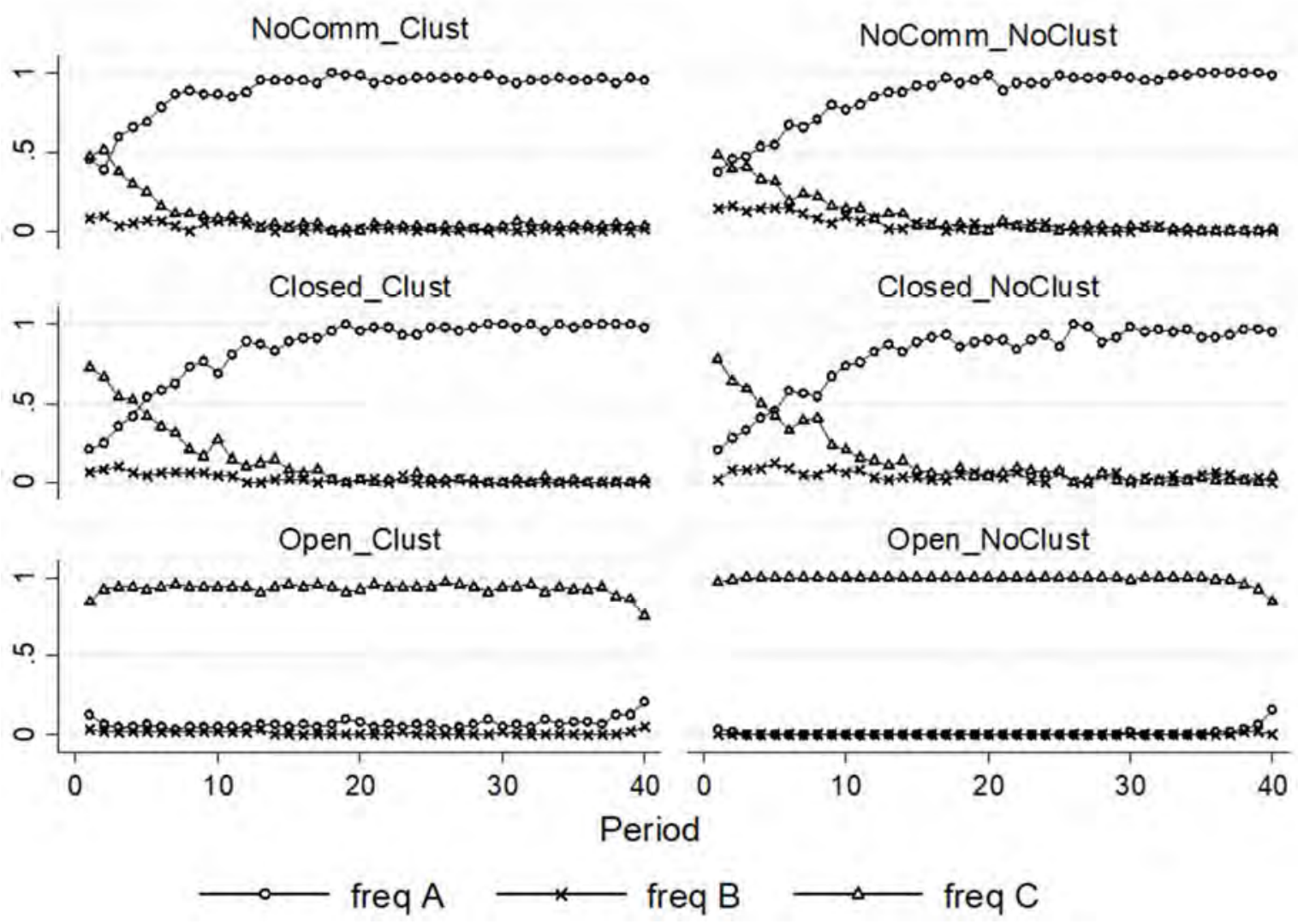

Given the strong differences in actions chosen, it is worth looking at average payoffs next. Without communication, people play A almost exclusively, with some losses due to a lack of coordination, so the average payoffs of 67.61 (clustered network) and 67.49 (non-clustered network) are not surprising and very close to the payoff of 70 per person in case of the (A,A)outcome. Closed communication increases the respective average payoffs in the network setting just very slightly, to 68.86 and 68.54. Payoffs increase significantly, to 94.14 in Clust and 99.32 in NoClust, only with open communication. ${ }^{14}$

\footnotetext{
${ }^{14}$ Ranksum two-tailed tests - using a matching group's (16 subjects) average over 40 periods as unit of observation gives $Z=2.309$ and 2.323, $p=0.021$ and 0.020 , for the respective differences in profits between NoComm_Clust and Open_Clust and between NoComm_NoClust and Open_NoClust; pooling across clustering conditions gives $Z=3.363$, $p=0.001$. Similarly, these tests give $Z=2.121$ and $2.323, p=0.034$ and 0.020 , for the respective differences in profits between Closed_Clust and Open_Clust and between Closed_NoClust and Open_NoClust; pooling across clustering conditions gives $Z=3.243$, $p=0.001$. The difference between Open_Clust and Open_NoClust is also significant ( $Z$ $=2.033, p=0.042)$. Neither of the differences between NoComm_Clust and Closed_Clust and between
} 
This is due to the overwhelming frequency of C-play, even though playing $\mathrm{C}$ bears the risk of no payoff if the matched player plays A or B. Yet, coordination seems to work well. In order to see this, we can consider the likelihood of successful coordination on (A,A), (B,B), or (C,C). It is not straightforward to define coordination in our framework; whether it should be at the pair level - but pairs are randomly chosen -, at the neighbors level, at the network-group level, or at the matching-group level. In Table 3, we show the rate of coordination at both the network-group level (reflecting the conservative assumption that a network group is coordinated if and only if all eight participants play the same action) and the level of the realized action-pairs.

Table 3: Frequencies of coordination on outcomes, by treatment

\begin{tabular}{llcccc}
\hline \hline & & \multicolumn{4}{c}{ Coordination at the network level: } \\
Treatment & Total & on A & on B & on C & Obs. \\
NoComm_Clust & 0.556 & 0.556 & 0.000 & 0.000 & 320 \\
NoComm_NoClust & 0.563 & 0.563 & 0.000 & 0.000 & 320 \\
Closed_Clust & 0.583 & 0.583 & 0.000 & 0.000 & 240 \\
Closed_NoClust & 0.503 & 0.503 & 0.000 & 0.000 & 320 \\
Open_Clust & 0.669 & 0.000 & 0.000 & 0.669 & 320 \\
Open_NoClust & 0.956 & 0.000 & 0.000 & 0.956 & 320 \\
\hline & & & Coordination at the pair level & \\
Treatment & Total & on A & on B & on C & Obs. \\
NoComm_Clust & 0.825 & 0.805 & 0.000 & 0.020 & 1280 \\
NoComm_NoClust & 0.819 & 0.779 & 0.005 & 0.035 & 1280 \\
Closed_Clust & 0.821 & 0.759 & 0.002 & 0.059 & 960 \\
Closed_NoClust & 0.794 & 0.712 & 0.009 & 0.073 & 1280 \\
Open_Clust & 0.877 & 0.015 & 0.000 & 0.862 & 1280 \\
Open_NoClust & 0.987 & 0.002 & 0.000 & 0.984 & 1280 \\
\hline \hline
\end{tabular}

NoComm_NoClust and Closed_NoClust is statistically-significant $(Z=1.062, p=0.289$ and $Z=1.443, p=0.149$ for the respective tests), although the pooled difference is in fact significant $(Z=2.199, p=0.028)$. No other comparison is at all close to statistical significance. 
The rate of complete coordination on A in the no- and closed-communication treatments ranges from 50-58\%, while the coordination rate at the realized-pair level ranges from $79-83 \%$; there is little difference across treatments or clustering conditions. No consensus coordination (i.e., on the network level) on B or C was ever observed without open communication treatments, although there is a tiny amount (less than 1\%) of pairwise coordination on B and a small amount (2-7\%) of pairwise coordination on C. Matters are very different with open communication, where there is a high rate of consensus coordination on C, remarkably so in the no-cluster case (with 95.6\%). It is clear from these findings that only open communication seriously affects behavior, so we summarize our findings on actions played in our first result.

Result 1. There is a very high likelihood of cooperative C-play with open communication, while the safe play (of A) is chosen overwhelmingly both without communication and with closed communication, particularly after the first few periods. The payoff-dominant equilibrium of $(B, B)$ is almost never played. There is no significant difference in play across the no-communication and closed-communication conditions.

\subsection{Analysis of messages}

\section{A. Diffusion of messages}

We begin the analysis of the diffusion of messages by showing first that receiving a C-message affects the likelihood of sending a C-message, meaning that C-messages are propagated through the network. We then show that this propagation depends on clustering, which then leads to differences in the distribution of C-messages between clustered and non-clustered networks.

We estimate the probability of sending the first C-message in a given time interval (i.e., seconds) for subjects that have already received at least one C-message in previous time intervals $\left(P_{R}\right)$ and for those that have not yet received any C-message $\left(P_{N R}\right)$. In Table 4 we report these estimates for different subset of periods, since the probability of sending a C-message could be affected by the experience gained in the repetition of the game. For example, groups that are well coordinated on action C could send fewer messages, and groups where some deviations happen could send more C-messages. In fact, we show below that there is a downward trend across the 40 periods in the likelihood of sending a message (see footnote 16 below). Thus we believe that the first period is the best for properly analyzing the propagation of messages. From the upper part of Table 4 we note that the probability of sending a first C-message is significantly higher 
when subjects have already received a C-message than when they haven't. This holds true for the first period and also for the first five periods (but not over all 40 periods).

Table 4: Estimation of sending a first C-message with open communication, conditional on having received a $C$-message $\left(P_{R}\right)$ or not $\left(P_{N R}\right)$

\begin{tabular}{lllll}
\hline \hline & & Period $=1$ & Period $<6$ & All Periods \\
Full Sample & $P_{R}$ & $0.043^{* * *}$ & $0.042^{* * *}$ & 0.018 \\
& $P_{N R}$ & 0.010 & 0.022 & 0.029 \\
\hline Reduced & $P_{R}$ & $0.025^{* * *}$ & $0.039^{*}$ & $0.020^{*}$ \\
Sample & $P_{N R}$ & 0.011 & 0.021 & 0.016 \\
\hline \hline
\end{tabular}

Sign test (two tails) of differences between $\mathrm{P}_{\mathrm{R}}$ and $\mathrm{P}_{\mathrm{NR}}$, separately for full and reduced sample. Significance at ${ }^{* * *} \mathrm{p}<0.01,{ }^{* *} \mathrm{p}<0.05,{ }^{*} \mathrm{p}<0.1$

In the lower part of Table 4 we report the estimates for a reduced sample. Here we exclude the observations where all subjects that have not sent any C-message yet either i) have already received at least one $\mathrm{C}$-message or ii) have not yet received any $\mathrm{C}$-message. The reason of this sample reduction is to keep only comparable observations, i.e. those observations where there are subjects in the network group that have received a C-message and other subjects that have not. So, in these observations all subjects have waited the same time from the beginning of the chat without sending C-messages. In the observations without this heterogeneity, a bias is possible if the individual probabilities to send C-messages are heterogeneous. Then we could have an adverse selection. This reduced sample mitigates this potential problem, but yields the same main results: the likelihood to send a C-message is higher for subjects who receive one.

Another way of looking at diffusion is to examine whether the number of C-messages that one receives has an impact on how likely one is to send a final C-message. Table 5 shows that this is in fact true with clustered networks, where the likelihood to send a final C-message increases monotonically with the number of C-messages received.

Hence, there is some contagion present. In the non-clustered network, one's rate of sending a final C-message is fairly constant at around $70 \%$, compared to an overall likelihood of $60 \%$ in the clustered network. This difference in behavior perhaps provides a first clue regarding the clustering effect with open communication that we will examine in more detail below. 
Table 5: Rates of final sent C-message, by \# C-messages received (Open Comm)

\begin{tabular}{ccc}
\hline \hline \# C messages received & Network Clust & Network NoClust \\
\cline { 2 - 3 } 0 & .352 & .683 \\
1 & .605 & .684 \\
2 & .649 & .716 \\
3 & .731 & .670 \\
4 & .822 & .727 \\
Total & .607 & .694 \\
\hline \hline
\end{tabular}

Figure 4: Cumulative distributions of the \# of C-messages (network level), by treatment, and at the end of the communication stage
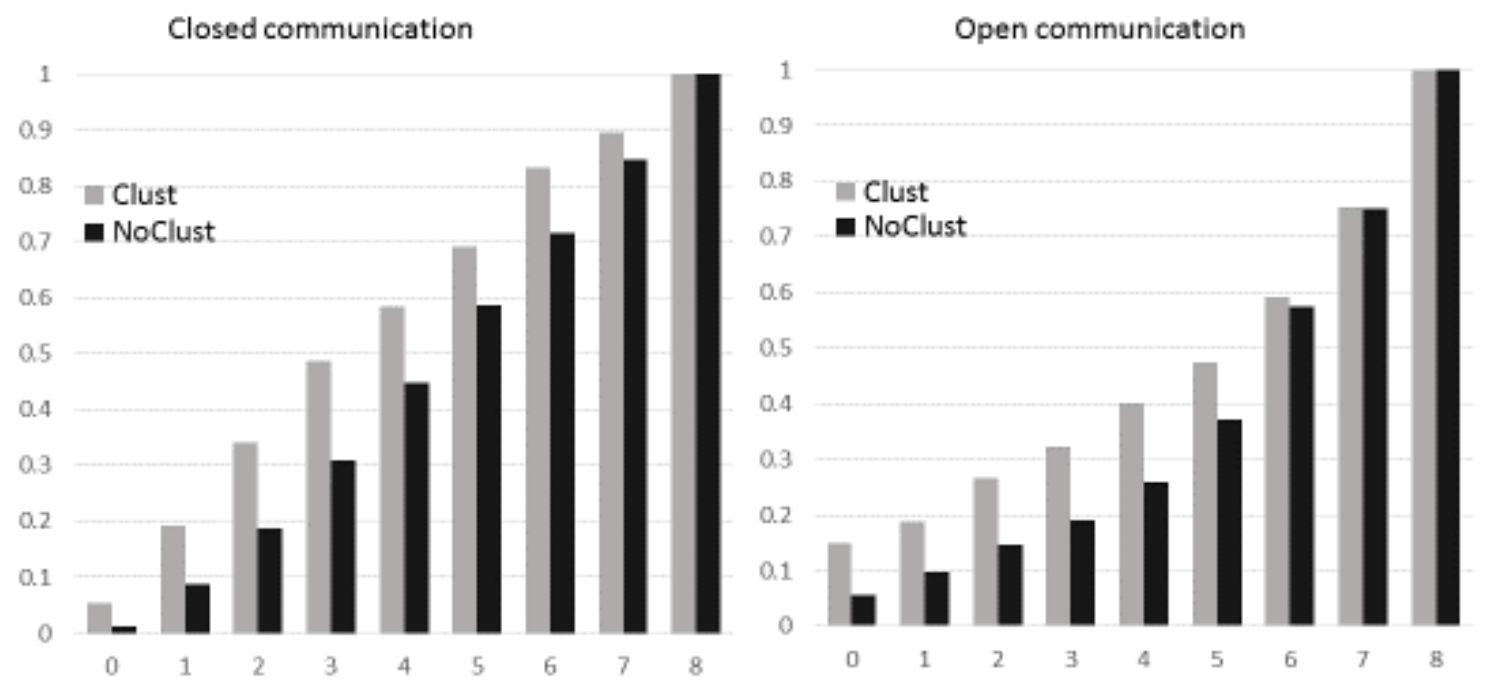

Finally, to test Hypothesis 1, we look at the network-group level and examine the distribution of final messages at the end of the communication phase of 90 seconds. This means that for each subject we consider the last message he or she sent before taking an action. Given the predominance of C-messages and our interest on the determinants of C-play, we focus on the 
distributions of C-messages. ${ }^{15}$ In Figure 4 we show the cumulative distribution: on the left for closed communication and on the right for open communication.

This gives us our second result, which supports our Hypothesis 1:

Result 2. The cumulative distribution in C-messages for the network without clusters (NoClust) first-order stochastically dominates (FOSD) that for the network with clusters (Clust). The same FOSD relationship is present in comparisons between open and closed communication.

\section{B. Messages and actions}

Which messages are actually the most credible in each of our treatments? Table 6 shows the messages sent and the subsequent play by the sender. Senders of A-messages played A 99.5\%, and $97.7 \%$ of the time in closed communication (see treatments Closed_Clust and Closed_NoClust). Thus, A-messages should be very, very credible. B-messages are not in the least credible in these treatments, since at most $15 \%$ of senders actually choose B after a Bmessage. C-messages are the most common by far in all treatments, but are not credible with closed communication. Senders of C-messages play C only around 25\% of the time with closed communication (26\% in Clust, and $24.6 \%$ in NoClust). In great contrast, C-messages are extremely credible with open communication, with sender consistency at $90 \%$ in Clust and nearly $100 \%$ in NoClust.

\footnotetext{
${ }^{15}$ In order to classify the content of messages in open communication, we let two coders work independently through all messages exchanged within a network and code them as suggestion to play action A, B, or C (recall that subjects could also choose not to send any message or a message that does not reveal which action the sender intends to play). The two coders provided very similar classifications (cross-coder consistency measured by Cronbach's alpha was very high, 0.9799). For closed communication, there was no need for coding as subjects entered either A, B, or C as their intended action or left the message space blank.
} 
Table 6: Messages and actions

\begin{tabular}{|c|c|c|c|c|c|c|}
\hline \multirow[b]{2}{*}{ Treatment } & \multirow[b]{2}{*}{ Message } & \multicolumn{4}{|c|}{ Sender Action } & \multirow[b]{2}{*}{ Consistency } \\
\hline & & Sent & $\mathbf{A}$ & B & C & \\
\hline \multirow[t]{4}{*}{ ClosedComm_Clust } & $\mathrm{A}$ & 392 & 390 & 1 & 1 & .995 \\
\hline & $\mathrm{B}$ & 143 & 130 & 12 & 1 & .084 \\
\hline & $\mathrm{C}$ & 941 & 669 & 26 & 246 & .261 \\
\hline & No & 444 & 436 & 3 & 5 & - \\
\hline \multirow[t]{4}{*}{ ClosedComm_NoClust } & A & 531 & 519 & 5 & 7 & .977 \\
\hline & B & 327 & 275 & 50 & 2 & .153 \\
\hline & $\mathrm{C}$ & 1538 & 1107 & 52 & 379 & .246 \\
\hline & No & 164 & 161 & 2 & 1 & - \\
\hline \multirow[t]{4}{*}{ OpenComm_Clust } & $\mathrm{A}$ & 5 & 0 & 0 & 5 & .000 \\
\hline & $\mathrm{B}$ & 2 & 0 & 0 & 2 & .000 \\
\hline & $\mathrm{C}$ & 1554 & 145 & 14 & 1395 & .898 \\
\hline & No & 999 & 26 & 7 & 966 & - \\
\hline \multirow[t]{4}{*}{ OpenComm_NoClust } & $\mathrm{A}$ & 6 & 1 & 1 & 4 & .167 \\
\hline & $\mathrm{B}$ & 4 & 0 & 0 & 4 & .000 \\
\hline & $\mathrm{C}$ & 1777 & 7 & 0 & 1770 & .996 \\
\hline & No & 773 & 14 & 1 & 758 & - \\
\hline
\end{tabular}

Note: “Consistency” means the likelihood that the sender's action and message are the same.

From Table 6 we can also see that, overall, messages were sent $75.2 \%$ of the time that they were feasible. In closed communication, messages were sent 76.9\% (Clust) and 93.6\% (NoClust) of the time, respectively. These frequencies are larger than in open communication where messages were sent $61.0 \%$ (Clust) and 69.8\% (NoClust) of the time, respectively. One immediate question is why more messages weren't sent with open communication. What we see in the 
analysis of the communication protocols is that groups appear to reach consensus quickly even without multiple messages in later rounds, so that not everyone needs to send a message. In fact, the percentage of people who send messages drops steadily over time in treatments Open_Clust and Open_NoClust, while there is no corresponding decrease in C-play. ${ }^{16}$

Result 3. Messages are sent more frequently with closed communication than with open communication. In the latter case, groups reach consensus quickly without multiple messages in later rounds, so that fewer messages are needed. The proportion of people who send messages drops steadily over time in treatments Open_Clust and Open_NoClust, while there is no corresponding decrease in C-play.

\subsection{Econometric analysis of the probability of playing action $\mathrm{C}$}

To organize and summarize all previous evidence we estimate the individual probability of playing action $\mathrm{C}$ using a logit model. In Table 7 we report the estimated marginal effects of 7 different specifications (full estimates are in Table C3 in Appendix C). We use the following explanatory variables:

- Period (from 1 to 40, by network),

- Net_NoClust (= 1 if the network is not clustered, 0 otherwise),

- Mess_C_rec (from 0 to 4; number of neighbors sending a C-message at the end of the communication stage),

- Mess_C_sent (= 1 if the subject sends a C-message at the end of the communication stage, 0 otherwise),

- Mess_C_rec \# Mess_C_sent (interaction of variables Mess_C_rec and Mess_C_sent), and

- Credibility (its specification is explained below).

\footnotetext{
${ }^{16}$ The likelihood that a participant sent a message was $77.7 \%, 63.3 \%, 53.9 \%$, and $49.1 \%$ in periods $1-10,11-20$, 2130, and 31-40, respectively, in treatment Open_Clust. The likelihood that a participant sent a message was $91.9 \%$, $74.5 \%, 61.9 \%$, and $51.9 \%$ in periods $1-10,11-20,21-30$, and 31-40, respectively, in treatment Open_NoClust. Recall that C-messages comprised more than $99.5 \%$ of all messages with open communication. The likelihood that a participant played C was $92.7 \%, 93.3 \%, 94.1 \%$, and $90.0 \%$ in periods $1-10,11-20,21-30$, and 31-40, respectively, in treatment Open_Clust. The likelihood that a participant played C was $99.5 \%, 100 \%, 99.8 \%$, and $96.9 \%$ in periods 110, 11-20, 21-30, and 31-40, respectively, in treatment Open_NoClust.
} 
In models (1)-(3) (one model for each communication protocol) we use only Period and Net_NoClust as explanatory variables. In models (4) and (5) we add the message variables (Mess_C_rec and Mess_C_sent and the interaction terms) to the estimation for the treatments with closed communication and open communication, respectively. In models (6) and (7) we finally add Credibility as an additional explanatory variable. We correct the standard errors at the matching group levels (and include dummies per matching groups, coefficients not reported).

Before proceeding to the estimation results, we explain how we construct our credibility variable. It is a function of a subject's past experiences on messages received and actions played by the paired opponent. Let $C R(t) \in[0,1]$ be the credibility of C-messages at period $t \geq 1$, i.e., the expected probability that a player is going to choose action $\mathrm{C}$ conditional on the fact that he sent message $\mathrm{C}$. Then the credibility that subject $i$ ascribes to a C-message evolves according to the following dynamics:

$$
C R_{i}(t+1)=\gamma \cdot C R_{i}(t)+(1-\gamma) \cdot\left(\left(1-p_{i}(t)\right) \cdot C R_{i}(t)+p_{i}(t) \cdot I_{i}(t)\right),
$$

where $p_{i}(t)=n_{i}(t) / 4$ and $n_{i}(t)$ be the number of C-messages received by $i$ at period $\mathrm{t}, I_{i}(t)$ is an indicator function that takes value 1 if the (randomly chosen) partner of player $i$ chose action $\mathrm{C}$ at $\mathrm{t}(0$ otherwise) and $\gamma \in[0,1]$ is the inertia parameter. This last parameter measures the weight of the prior on the credibility of messages. Then $(1-\gamma)$ is the weight assigned to the new experience.

The new experience of a round is used as follows: The idea is that subject $i$, in order to assign a credibility to a C-message, looks at the number $n_{i}(t)$ of $\mathrm{C}$-messages. Then he knows that with probability $\left(1-p_{i}(t)\right)$ he is matched with a player that did not send a C-message. Thus, with such a probability the action chosen by his opponent should not affect the credibility of C-messages (and, hence, the posterior equals the prior). On the contrary, with probability $p_{i}(t)$ he is matched with a player that did send a C-message. Thus, with such a probability the action chosen by his opponent should affect the credibility of C-messages ( 1 if the opponent chose action $\mathrm{C}$, and 0 otherwise). Note that this variable is a dynamic measure of credibility and the result of Proposition 2 in section 3.2 provides a rationale for the credibility levels of messages in the first periods (when experience is absent or small).

In the estimation we have repeated the logit models (6) and (7) ten times, one for each possible value of $\gamma \in\{0,0.1,0.2, \ldots, 1\}$. We did so for closed communication (model 6 ) and for 
open communication (model 7). In each case we have chosen the value of $\gamma$ that provides a better estimation (by looking at the value that maximizes the likelihood function). Then we report in Table 7 the estimations using the credibility variable with such values of $\gamma$ ( $\gamma=0.7$ with closed communication and $\gamma=0.4$ with open communication).

Table 7: Marginal effects on the probability of playing C

(evaluated at Period 20 and means of other variables unless otherwise specified)

\begin{tabular}{|c|c|c|c|c|c|c|c|}
\hline \multirow{3}{*}{ variables } & (1) & (2) & (3) & (4) & (5) & (6) & (7) \\
\hline & No Comm & Closed & Open & Closed & Open & Closed & Open \\
\hline & & Comm & Comm & Comm & Comm & Comm & Comm \\
\hline Period & $-0.009 * * *$ & $-0.015 * * *$ & -0.000 & $-0.007 * * *$ & -0.000 & -0.003 & -0.0002 \\
\hline at Net_NoClust $=0$ & $(0.002)$ & $(0.001)$ & $(0.000)$ & $(0.001)$ & $(0.000)$ & $(0.002)$ & (0.0004) \\
\hline Period & $-0.010^{* * *}$ & $-0.016 * * *$ & $-0.004 * * *$ & $-0.008^{* * *}$ & $-0.004^{* * *}$ & $-0.003 * * *$ & $-0.004^{* * *}$ \\
\hline at Net_NoClust=1 & $(0.001)$ & $(0.002)$ & $(0.001)$ & $(0.001)$ & $(0.001)$ & $(0.001)$ & $(0.001)$ \\
\hline \multirow{2}{*}{ Net_NoClust } & $-0.046^{* *}$ & $0.038^{* *}$ & $0.025^{* * *}$ & 0.001 & $0.022 * *$ & -0.004 & $0.021^{* *}$ \\
\hline & $(0.022)$ & (0.019) & $(0.010)$ & $(0.020)$ & $(0.011)$ & $(0.018)$ & $(0.010)$ \\
\hline Mess_C_rec & & & & 0.001 & 0.003 & 0.001 & 0.005 \\
\hline At Mess_C_sent $=0$ & & & & $(0.002)$ & $(0.011)$ & $(0.004)$ & $(0.011)$ \\
\hline Mess_C_rec & & & & $0.070^{* * *}$ & $0.010^{* * *}$ & $0.073^{* * *}$ & $0.011^{* * *}$ \\
\hline at Mess_C_sent $=1$ & & & & $(0.007)$ & $(0.004)$ & $(0.005)$ & $(0.004)$ \\
\hline Mess_C_sent & & & & 0.004 & 0.004 & 0.005 & 0.004 \\
\hline at Mess_C_rec $=0$ & & & & $(0.005)$ & $(0.032)$ & $(0.006)$ & $(0.029)$ \\
\hline Mess_C_sent & & & & $0.049 * * *$ & 0.026 & $0.059^{* * *}$ & 0.023 \\
\hline at Mess_C_rec $=2$ & & & & $(0.012)$ & $(0.020)$ & $(0.008)$ & $(0.018)$ \\
\hline Mess_C_sent & & & & $0.239 * * *$ & $0.038 * * *$ & $0.243^{* * *}$ & $0.032 * *$ \\
\hline at Mess_C_rec $=4$ & & & & $(0.027)$ & $(0.014)$ & $(0.021)$ & $(0.013)$ \\
\hline Credibility & & & & & & $0.178^{* * *}$ & \\
\hline$(\gamma=0.7)$ & & & & & & $(0.052)$ & \\
\hline Credibility & & & & & & & $0.066 * * *$ \\
\hline$(\gamma=0.4)$ & & & & & & & $(0.016)$ \\
\hline
\end{tabular}

Robust standard errors in parentheses ${ }^{* * *} p<0.01,{ }^{* *} p<0.05,{ }^{*} p<0.1$

Turning to the estimation results in Table 7, we note that without communication, action $\mathrm{C}$ is played with a significantly larger probability in network Clust. This result is consistent with 
previous theoretical and experimental studies on networks without communication, where clustering has a positive effect on cooperation. However, this clustering effect turns negative and significant in all specification of open communication, which is consistent with our Hypothesis 3. It seems that in our game and communication structure within networks, the direct positive effect of clustering on cooperation is dominated by the indirect, but negative, effect of clustering via messages' diffusion. When closed communication is used, there is no significant clustering effect (see columns 4 and 6).

We summarize these findings as follows:

Result 4. With open communication, C-play is more frequent without clustering than with positive clustering. For closed communication we find no network effect once we add control variables. Without communication, clustering has a positive impact on the rate of C-play, which confirms previous works.

Looking at the effect of messages, we see that there is a positive effect on cooperation of the number of C-messages received only when a C-message was sent. This effect is displayed in both protocols of communication and all specifications (see columns 4 and 5); perhaps surprisingly, it is significantly larger in closed communication. Moreover, we find a positive effect on cooperation of the sent C-message: for a subject that sent a C-message, the probability of playing $C$ is significantly higher compared to a subject that did not send a C-message. But we note that this effect is conditional on having received some C-message from the neighbors. Again, this effect seems to (surprisingly) be larger in closed communication. Finally, we find a significant positive effect of the credibility variable in both communication settings and this effect is larger with closed communication.

Therefore, given that the positive effects of messages are larger for closed communication, a natural question is: Why does cooperation collapse in closed communication, but not in open communication? Figure 5 provides useful evidence for answering this question. It shows the evolution of credibility, both in closed communication (upper panel) and open communication (lower panel). 


\section{Figure 5: Evolution of average credibility by treatment and period}

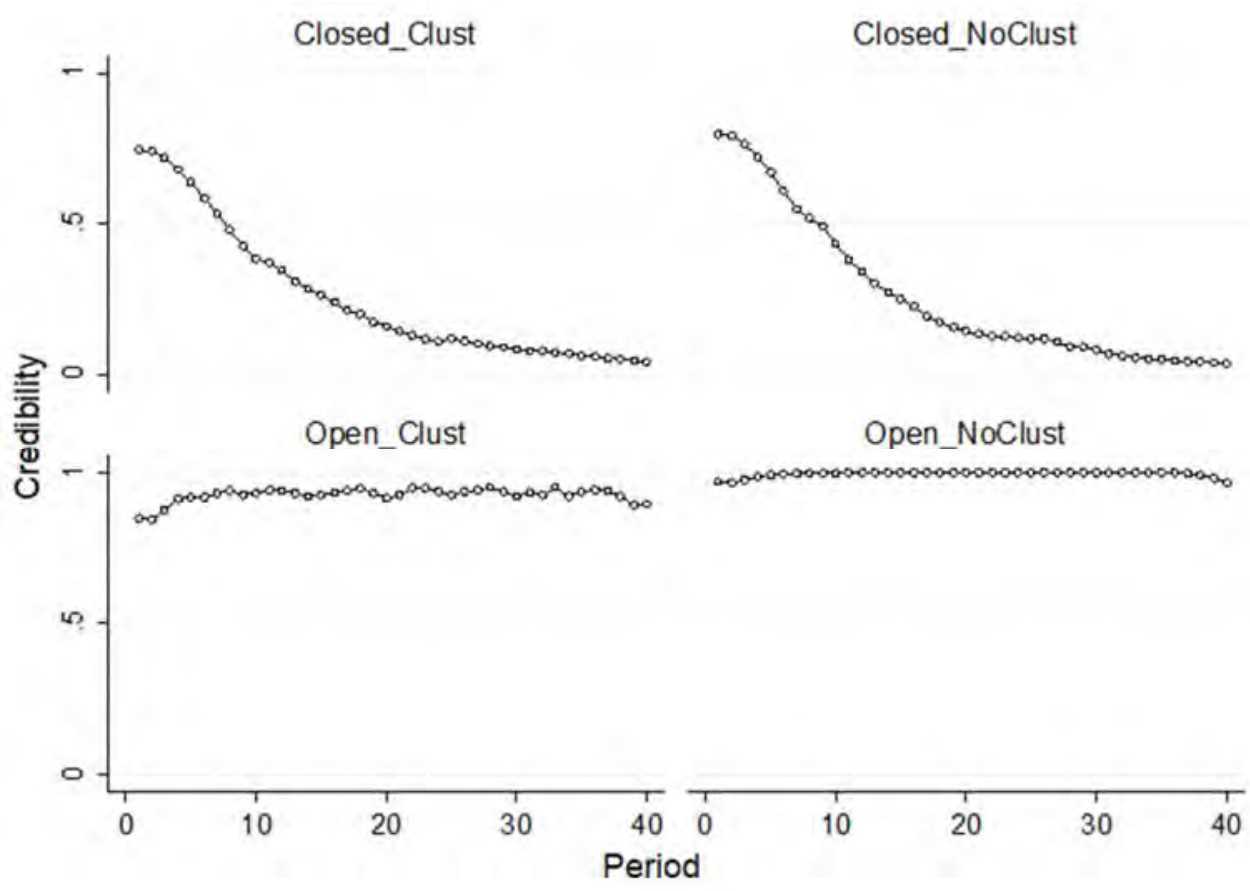

The credibility of $\mathrm{C}$ messages in the closed-communication treatments converges to zero and drops below $50 \%$ before period 10 . Hence, while messages would work better in closed communication (if credible), the quick drop for credibility with closed communication also allows C-play to erode quickly, thus driving the network members to play A (see also Figure 4). The situation is completely different with open communication where credibility stays close to $100 \%$ throughout the entire experiment. This marked difference between closed and open communication strongly supports our Hypothesis 1, leading to Result 5.

A rationale for this result can be found in our model of credibility (section 3.2). Under the assumption of high lying costs in open communication, the dominant strategy is to play according to the sent message. This explains the stability of our credibility measure at very high rates during the 40 periods of play. On the contrary, if lying costs are not high enough (in closed communication), responding in a different manner can pay for some beliefs.

Result 5. The credibility of messages is much higher with open communication than with closed communication, leading to the very high rates of C-play with chat. 


\subsection{A robustness check with bi-lateral communication}

One hypothesis about the failure of cooperation on C-play and lower credibility of Cmessages with closed communication is that the network environment introduces some level of uncertainty in the behavior of the matched player: this uncertainty is due to the unseen messages received by neighbors and the player with whom one is matched in the game. In order to remove this uncertainty we run a control treatment with closed communication involving bi-lateral matching rather than the network format. Our hypothesis is that the uncertainty characterizing the network environment is an obstacle to efficient selection, for which reason we should observe more C-play in the setting with bi-lateral matching. In fact, we find support for our conjecture, since we see an overall rate of C play of 30.5\% with bi-lateral matching. Two-tailed ranksum tests with session-level data show that this rate is higher than the rate for both clustered networks (13.2\%) and non-clustered networks (15.2\%) with closed communication $(Z=2.121, p=0.034$ and $Z=1.732, p=0.083$, respectively).

However, C-play in the bi-lateral treatment is nevertheless still significantly lower than with open communication $(\mathrm{Z}=2.309, p=0.021, Z=2.323 p=0.021$ for respective comparisons to treatments Open_Clust and Open_NoClust). So even though a simpler environment helps to increase efficiency with closed communication, it does not come close to the level of efficiency made possible by open communication in the original complex network environment. Figure 6 shows patterns for treatment Closed_Bilateral that are similar to the ones for treatments Closed_Clust and Closed_NoClust, but the average frequency of C play is significantly higher (and twice as high) with bi-lateral closed communication with respect to closed communication in networks, especially with respect to the clustered network (compare Figure 6 and Figure 4). Nevertheless, although initially the frequency of C play is around $80 \%$ in the first round (compared to around 50\% in the network settings) and the decline is slower than in treatments Closed_Clust and Closed_NoClust, it progressively declines to levels close to zero at period 30. The results suggest that indeed it is easier to achieve cooperation on playing $\mathrm{C}$ in the bi-lateral treatment early on, although we see the same collapse of $\mathrm{C}$ choices over time that we saw in our network treatments with closed communication. So, the only stable mechanism to achieve almost full efficiency in our demanding game was open communication. 
Figure 6: Relative frequencies in closed communication with bi-lateral interaction

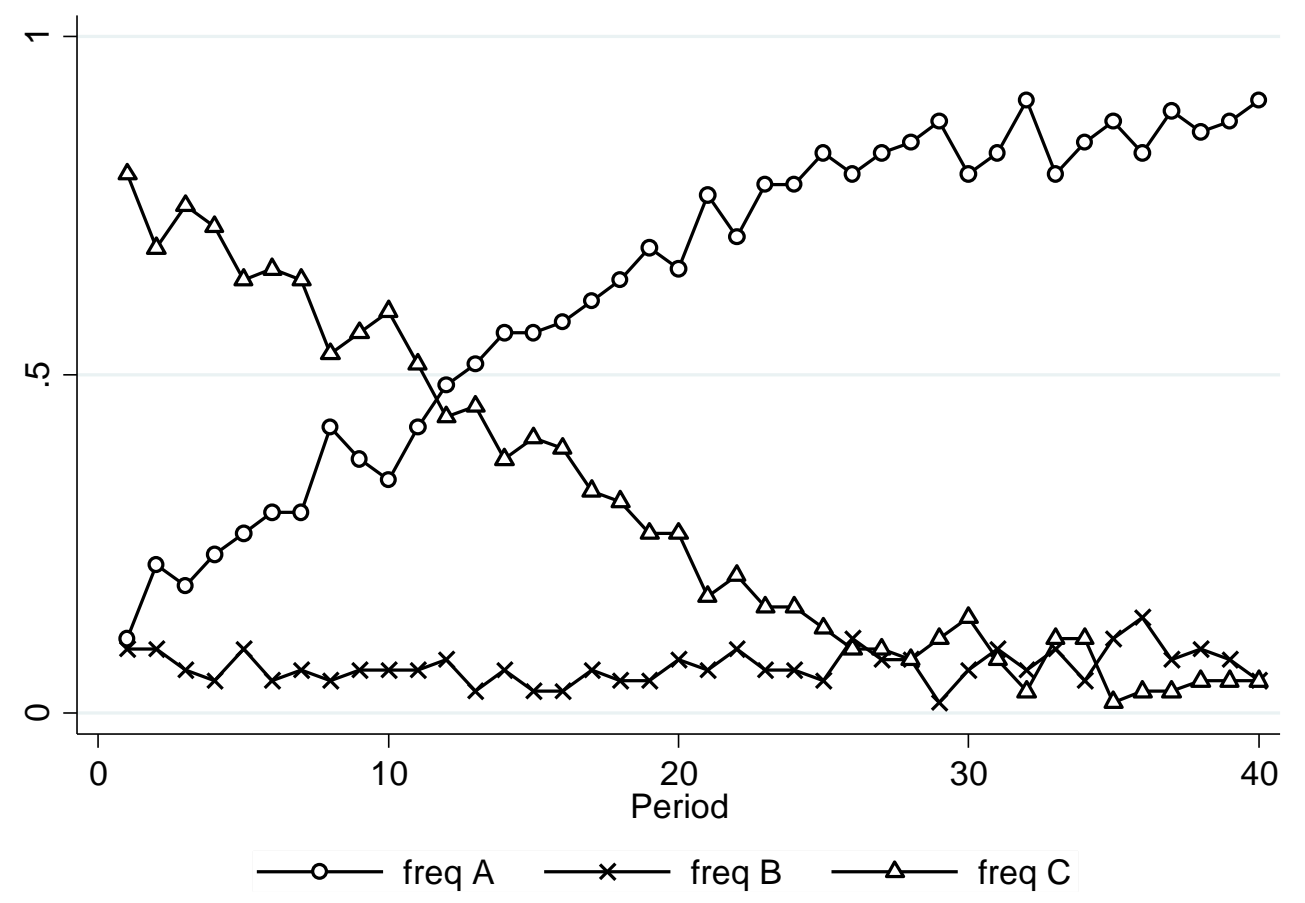

\subsection{A further robustness check with a standard Stag Hunt game}

We had formed our game in Figure 1 by adding a row and a column to the standard Stag Hunt game shown in Figure 7, which Charness (2000) tested in a bi-lateral setting with and without closed communication.

Figure 7: The standard Stag Hunt game

\section{Player 2}

A B

Player 1

A

70,70

80,0
B
0, 80
90, 90 
Both $(A, A)$ and $(B, B)$ are pure-strategy equilibria in the standard game. We conducted treatments with and without clustered networks and with and without closed communication to check whether there was anything odd in our subject pool. The implementation of the sessions was the same as with the extended Stag Hunt, except that we dropped strategy C. Table 8 shows the treatments with the standard Stag Hunt. ${ }^{17}$ We had 368 new participants in these sessions.

Table 8: Treatment, sessions and participants in the standard Stag Hunt

\begin{tabular}{lcc}
\hline \hline & Sessions & Participants \\
No communication, clustered network & 2 & 64 \\
No communication, non-clustered network & 2 & 64 \\
Closed communication, clustered network & 4 & 112 \\
Closed communication, non-clustered network & 4 & 128 \\
\hline \hline
\end{tabular}

In Charness (2000), the rate of B play with (without) communication was 90\% (29\%) over 10 periods. Given the finding above that social efficiency is easier to achieve in a bi-lateral setting (see treatment Closed_Bilateral), we expected that closed communication be somewhat less effective in our network settings. In fact, this is the case, although not dramatically so, as Table 9 shows. The rate of B play with closed communication and with (without) clustering is $77.12 \%$ (75.12\%), which is far higher than in the 3-option extended Stag Hunt. By contrast, the rate of B play with no communication and with (without) clustering is $11.45 \%$ (13.01), not very different than in the 3-option game. The results without communication are somewhat similar to those in the main experiment, in that the safe choice, $\mathrm{A}$, was made at nearly the identical rates and so riskier choices (everything else) are also made at nearly the identical rates.

\footnotetext{
${ }^{17}$ Given the tremendously high rates of cooperation we had already observed in the extended Stag Hunt, it seemed superfluous to have open communication in the far simpler standard Stag Hunt.
} 
Table 9: Frequencies of actions $A$ and $B$ in treatments with standard Stag Hunt game.

\begin{tabular}{ccccc}
\hline \hline & \multicolumn{2}{c}{ No Communication } & \multicolumn{2}{c}{ Closed Communication } \\
& Clust & NoClust & Clust & NoClust \\
\cline { 2 - 5 } $\mathrm{A}$ & $88.55 \%$ & $86.99 \%$ & $22.88 \%$ & $24.88 \%$ \\
& $(2267)$ & $(2227)$ & $(1025)$ & $(1274)$ \\
$\mathrm{B}$ & $11.45 \%$ & $13.01 \%$ & $77.12 \%$ & $75.12 \%$ \\
& $(293)$ & $(333)$ & $(3455)$ & $(3846)$ \\
\multirow{3}{*}{ Total } & $100 \%$ & $100 \%$ & $100 \%$ & $100 \%$ \\
& $(2560)$ & $(2560)$ & $(4480)$ & $(5120)$ \\
\hline \hline
\end{tabular}

The bottom number in each cell (in parentheses) is the number of observations.

Figure 8 shows the choices made over time. We see that the rate of B-play starts out at around $50 \%$ in the first periods of the no-communication treatments, but then quickly drops to almost nothing (less than 1\%) in the final 10 periods. With closed communication, B is played at the rate of $84 \%$ in the first 10 periods, which drops to $68 \%$ in the final 10 periods; the patterns are quite similar across network types. Looking at the time trend, it might seem that behavior is becoming completely mixed, but this is not actually the case. Instead, some groups converge to B play while other groups converge to A play (see Table C4 and Figure C2 in Appendix C for results by 16-person matching group). The actual rate of mis-coordination in these sessions was only $12.8 \%$ (and was only $12.3 \%$ in the last 10 periods). ${ }^{18}$ Overall, participants coordinated on A $17.7 \%$ of the time and coordinated on B $69.5 \%$ of the time.

Given the 60-percentage-point differences in rates of $\mathrm{B}$ play across communication treatments in Table 9, it should be clear that closed communication is quite effective in our network environment in the standard Stag Hunt game. Statistically, with clustered networks, every 16person group had a higher rate of B play than did any such group in the no-communication treatment and the same was true with non-clustered networks. The Wilcoxon ranksum test

\footnotetext{
${ }^{18}$ If behavior were completely random (50\% and uncorrelated), we should see mis-coordination at the realized pair level half of the time. Even a rate of (uncorrelated) B play of $75 \%$ would lead to mis-coordination $37.5 \%$ of the time. However, the actual rate of mis-coordination in these sessions was much lower at around $13 \%$.
} 
indicates the difference across communication treatments is significant at $p=0.002$ for clustered networks and also for non-clustered networks. Note that $(B, B)$ is the preferred equilibrium in both the 3-choice game and the "standard" Stag Hunt. However, while closed communication is rather effective in the standard Stag Hunt even in our network setting, it fails to prevent a collapse to Aplay there in our 3-choice game. Without the temptation of a non-equilibrium choice with higher payoffs, a signal of B is sufficiently credible ( $84.9 \%$ of those sending message B play action B, with $84.2 \%$ in Clu and $85.6 \%$ in NoClu) to sustain predominant B-play in the standard Stag Hunt.

Figure 8: Standard Stag Hunt - Average relative frequency of action B across periods

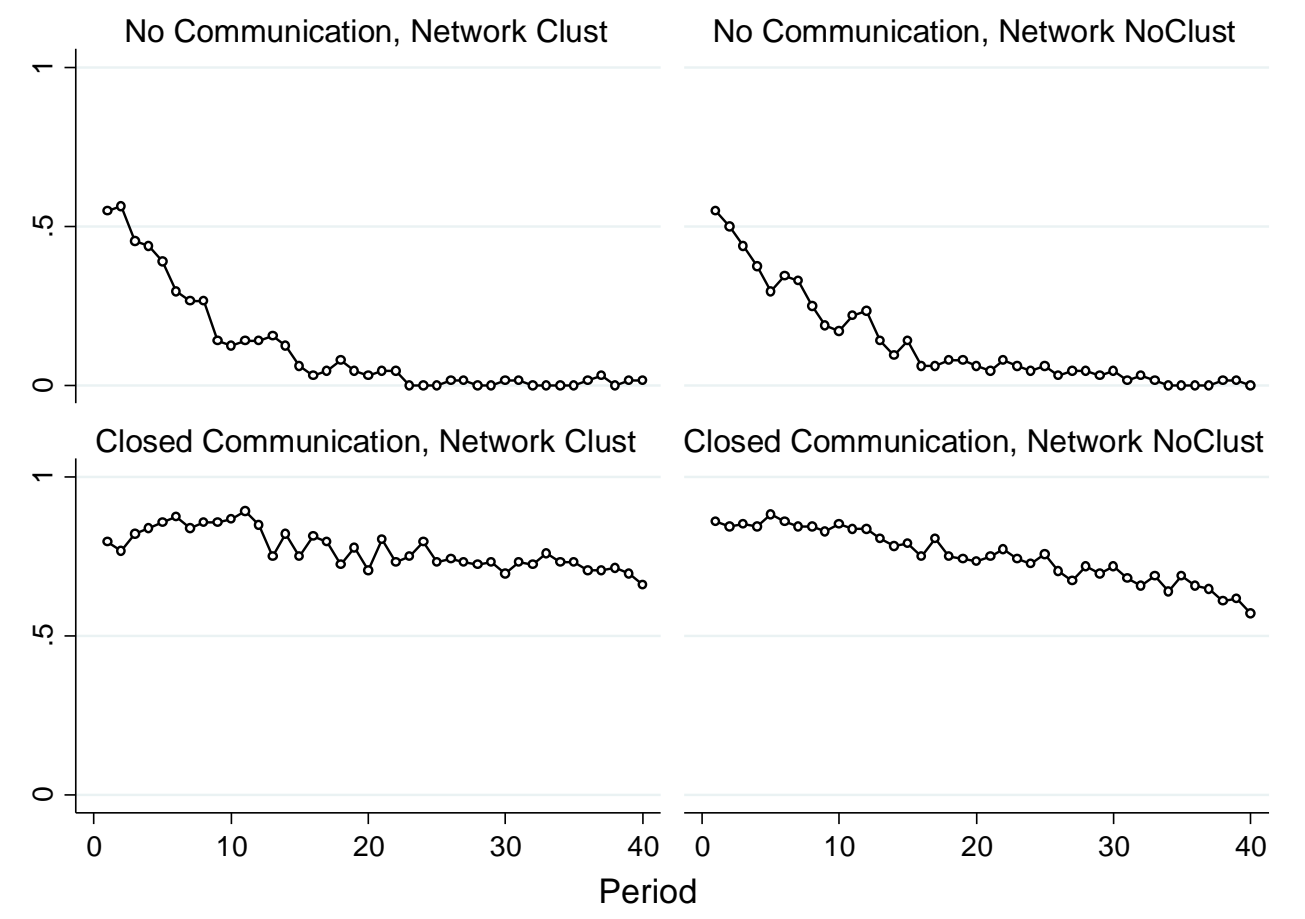




\section{Conclusion}

We investigate behavior in a novel experimental game played in a network setting. We vary two dimensions: 1) the type of communication that is feasible and 2) the degree of clustering in the network. No previous paper has considered this combination. The network setting implies that individuals can only interact with a subset of other people in the network (and, of course, in the overall population). Our game features two pure-strategy Nash equilibria and a sociallyefficient, but non-equilibrium, outcome.

We find major differences in behavior across communication technologies. In fact, previous experimental work on networks has almost completely ignored the role of communication, which seems odd in a world where communication is highly prevalent. Our results show that without communication, there is some initial optimism but play rapidly devolves to the safe risk-dominant action, with a rate of $96.4 \%$ in the last 20 of the 40 periods. There is little difference with closed communication, where only a message indicating (without words) the intended action can be transmitted; the rate of the risk-dominant play is $95.6 \%$ in the last 20 periods. However, matters change dramatically with open communication (chat with those people with whom one is linked), where the socially-efficient play is observed nearly all of the time (95.2\%) in the last 20 periods and the risk-dominant play is hardly ever (4.5\%) observed in the last 20 periods. The degree of effectiveness for open communication is remarkable, particularly since the network environment is a much more difficult one for successful coordination and cooperation (as we see in a robustness test).

Regarding the effect of clustering, theoretical and experimental work that does not incorporate communication has indicated that a higher degree of clustering leads to more efficient outcomes. We do confirm this result without communication, but we find that open communication reverses the direction of the effect. There is a moderate-yet-significant difference across clustering with open communication; further, the difference of the effect of clustering across no communication and open communication is larger and highly significant. There is a trade-off between having more repeated (and presumably deeper) interaction with the same people (with positive clustering) and reaching more individuals less frequently (with zero clustering). The first seems to be more useful when communication is not feasible, but the latter is more important when rich communication is a feature of the environment. 
We develop a theoretical model that addresses the issue of diffusion of messages across the network, given that there is duration to the communication phase in our game (and in the basic Stag hunt game that is used as a robustness check); we are the first to consider this factor. In fact, the prediction is that diffusion will be more rapid without clustering, which is consistent with our data. A second theoretical model considers credibility and once again our data support the model's conclusion that credibility will be higher with open communication than with closed communication. So our experimental and theoretical evidence shows that clustering will not necessarily lead to more efficient play when free-form communication is possible.

We have really only established a beachhead in terms of advancing the issue of how different forms of communication combine with different network characteristics. Given the fact that communication is ubiquitous in the field and that network interaction is quite common, we feel that this is an important area for future research. We hope that others join us in this quest. 


\section{References}

Abeler, J., Nosenzo, D., Raymond, C. (2019). Preferences for truth-telling. Econometrica, forthcoming.

Alós-Ferrer, C., Kirchsteiger, G., Walzl, M. (2010). On the evolution of market institutions - The platform design paradox, Economic Journal, 120 (543), 215-243.

Assenza, S., Gómez-Gardeñes, J., Latora, V. (2008). Enhancement of cooperation in highly clustered scale-free networks, Physical Review E, 78, 017101.

Aumann, R. (1990). Nash-equilibria are not self-enforcing, in Economic Decision Making: Games, Econometrics and Optimisation (J. Gabszewicz, J.-F. Richard, and L. Wolsey, Eds.), pp. 201206. Amsterdam: North-Holland.

Babcock, P., Bedard, K., Charness, G., Hartman, J., Royer, H. (2015). Letting down the team? Social effects of team incentives, Journal of the European Economic Association 13(5), 841870.

Ben-Ner, A., Putterman, L., Ren, T. (2011). Lavish returns on cheap talk: Two-way communication in trust games, Journal of Socio-Economics 40(1), 1-13.

Ben-Porath, E., Dekel, E. (1992). Signaling future actions and the potential for sacrifice, Journal of Economic Theory 57, 36-51.

Berninghaus, S. K., Erhart, K.-M., Keser, C. (2002). Conventions and local interaction structures: Experimental evidence, Games and Economic Behavior 39(2), 177-205.

Blume, A., DeJong, D., Kim, Y.-G., Sprinkle, G. (1998). Experimental evidence on the evolution of meaning of messages in sender-receiver games, American Economic Review 88(5), 13231340.

Blume, A., Ortmann, A. (2007). The effects of costless pre-play communication: Experimental evidence from games with Pareto-ranked equilibria, Journal of Economic Theory 132(1), 274290.

Brandts, J., Ellman, M., Charness, G. (2016). Let's talk: How communication affects contract design, Journal of the European Economic Association 14(4), 943-974.

Brosig, J., Weimann, J., Ockenfels, A. (2003). The effect of communication media on cooperation. German Economic Review 4(2), 217-241.

Cassar, A. (2007). Coordination and cooperation in local, random and small world networks: Experimental evidence, Games and Economic Behavior 58(2), 209-230. 
Charness, G. (2000). Self-serving cheap talk: A test of Aumann's conjecture, Games and Economic Behavior 33(2), 177-194.

Charness, G., Dufwenberg, M. (2006). Promises and partnership, Econometrica 74(6), 1579-1601.

Charness, G., Dufwenberg, M. (2011). Participation, American Economic Review 101(4), 12131239.

Charness, G., Feri, F., Meléndez-Jiménez, M.A., Sutter, M. (2014). Experimental games on networks: Underpinnings of behavior and equilibrium selection, Econometrica 82(5), 16151670.

Choi, S., Lee, J. (2014). Communication, coordination and networks, Journal of the European Economic Association 12(1), 223-247.

Clark, K., Kay, S., Sefton, M. (2001). When are Nash equilibria self-enforcing? An experimental analysis, International Journal of Game Theory 29, 435-456.

Cooper, R.C., De Jong, D., Forsythe, R., Ross, T. (1992), Communication in coordination games, Quarterly Journal of Economics 107, 739-771.

Crawford, V. (1998), A survey of experiments on communication via cheap talk, Journal of Economic Theory 78, 286-298.

Dal Bó, P., Foster, A., Putterman, L. (2010). Institutions and behavior: Experimental evidence on the effects of democracy, American Economic Review 100(5), 2205-2229.

Dennis, A.R., Kinney, S.T., Hung, Y.-T.C. (1999). Gender differences in the effects of media richness, Small Group Research 30(4), 405-437.

Eshel, I., Samuelson, L., Shaked, A. (1998). Altruists, egoists and hooligans in a local interaction model, American Economic Review 88(1), 157-179.

Farrell, J. (1993). Meaning and credibility in cheap talk games, Games and Economic Behavior 5(4), 514-531.

Farrell, J., Rabin, M. (1996). Cheap talk, Journal of Economic Perspectives 10(3), 103-118.

Gneezy, U., Kajackaite, A., Sobel, J. (2018). Lying aversion and the size of the lie, American Economic Review 108(2), 419-453.

Holland, P.W., Leinhardt, S. (1971). Transitivity in structural models of small groups, Comparative Group Studies 2(2), 107-124.

Huck, S., Müller, W. (2005). Burning money and (pseudo) first-mover advantages: An experimental study on forward induction. Games and Economic Behavior 51, 109-127. 
Jackson, M.O. (2010). An Overview of Social Networks and Economic Applications, in The Handbook of Social Economics, ed. by J. Benhabib, A. Bisin, and M.O. Jackson. Amsterdam: North-Holland.

Kearns, M., Judd, S., Tan, J., Wortman, J. (2009). Behavioral experiments on biased voting in networks, Proceedings of the National Academy of Sciences of the United States of America 106(5), 1347-1352.

Keser, C., Erhart, K.-M., Berninghaus, S.K. (1998). Coordination and local interaction: Experimental evidence, Economics Letters 58(3), 269-275.

Kohlberg, E., Mertens, J.-F. (1986). On the strategic stability of equilibria, Econometrica 54, 1003-1038.

Matthews, S.A., Okuno-Fujiwara, M., Postlewaite, A. (1991). Refining cheap-talk equilibria, Journal of Economic Theory 55(2), 247-273.

Melamed, D., Harrell, A., Simpson, B. (2017). Cooperation, clustering, and assortative mixing in dynamic networks, Proceedings of the National Academy of Sciences of the United States of America 115(5), 951-956.

Parker, E. (2014). Social media and the Hong Kong protests, The New Yorker, retrieved from http://www.newyorker.com/tech/elements/social-media-hong-kong-protests.

Rabin, M. (1990). Communication between rational agents, Journal of Economic Theory 51(1), $144-170$.

Rockmann, K.W., Northcraft, G.B. (2018). To be or not to be trusted: The influence of media richness on defection and deception, Organizational Behavior and Human Decision Processes 107, 106-122

Rousseau, J-.J. $(1755,1988)$. Discourses on the origin and foundation of inequality among men. In A. Ritter and J. Conaway Bondanella (eds.), Rousseau's Political Writings, pp. 3-57. New York, Norton.

Sutter, M., Haigner, S., Kocher, M.G. (2010). Choosing the carrot or the stick? Endogenous institutional choice in social dilemma situations, The Review of Economic Studies 77(4), 1540-1566.

van Damme, E. (1989). Stable equilibria and forward induction, Journal Economic Theory 48, 476-496. 
Vega-Redondo, F. (2006). Building up social capital in a changing world, Journal of Economic Dynamics and Control 30(11), 2305-2338.

Vega-Redondo, F., Marsili, M., Slalina, F. (2005). Clustering, cooperation, and search in social networks, Journal of the European Economic Association 3(2-3), 628-638.

Watts, D.J., Strogatz, S.H. (1998). Collective dynamics of 'small-world' networks, Nature 393, $440-442$. 


\section{Appendix A: Experimental instructions}

In the following we report the instructions (translated into English) of treatment Open_Clust. Then, we indicate the differences of treatments Closed_Clust and NoComm_Clust. Treatments with network NoClust only differ in the network used. Treatments with the standard Stag-Hunt game (cf. Section 4.5) only differ on the fact that action $\mathrm{C}$ is not available.

\section{Instructions treatment Open_Clust}

The aim of this Experiment is to study how individuals make decisions in certain contexts. The instructions are simple. If you follow them carefully you will earn a non-negligible amount of money in cash (Euros) at the end of the experiment. During the experiment, your earnings will be accounted in ECU (Experimental Currency Units). Individual payments will remain private, as nobody will know the other participants' payments. Any communication among you is strictly forbidden and leads to exclusion from any payments.

1.- The experiment consists of 40 rounds. In each round you will be randomly assigned to a group of 8 participants. This group is determined randomly at the beginning of the round. Therefore, the group you are assigned to changes at each round. In this room, there are 16 participants (including yourself) that are potential members of your group. That is, at every round your group of 8 participants is selected from among these 16 participants, each of the others being equally likely to be in your group. You will not know the identities of any of these participants.

2.- At each round, you and each of the remaining seven participants of your group will be assigned one position of the following NETWORK. The positions in the network are numbered from 1 to 8. In the network, a link is represented by a line (connection) between two positions. Each position has four links. For example, position 4 is linked to positions 2, 3, 5 and 6 (but it is not linked to the remaining positions).

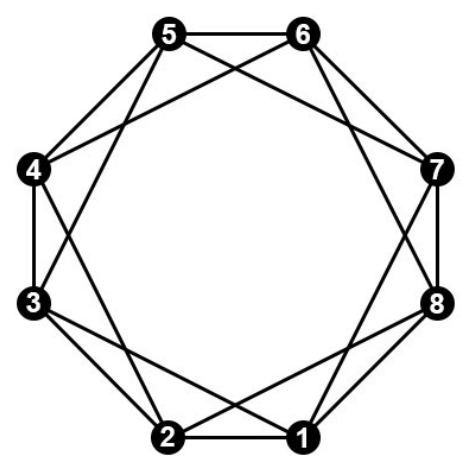


3.- Each round consists of two stages: first, communication stage, and second, action stage. Your final earnings will depend only on what you choose and what others in your group choose in the action stage. Three actions, A, B and C, are available in the action stage. The communication stage that precedes the action stage involves each participant sending messages. The messages are sent via chat in the communication stage. A message may indicate your intended action in the subsequent action stage. However, you do not have to follow your message when it comes to making an action choice. We now describe each of these two stages in more detail.

4.- Communication stage. This stage consists of 90 seconds in which each participant of the group can send messages to those ones he/she is linked to in the network. During this stage, at each point in time, the participants send messages via chat. The rules of the chat are that you can write whatever message you want, as far as the content of the message is not offensive (profanity is not allowed), and that you do not reveal your name or any personal identifying information. Noncompliance with the chat rules leads to exclusion from any payments. The messages sent by a participant will be immediately visible in the screens of all the participants linked to him/her in the network. Each participant can send as many messages as he/she wishes during this stage, and all of them will appear in the screens of the participants linked to him/her. When the time finishes, the participants can no longer send any new message.

For example, the participant in position 4 will observe the messages of the participants in positions 2, 3, 5 and 6 (and vice versa), but not those of participants in positions 1, 7 and 8 .

5.- Action stage. When the communication stage ends, each participant of your group will be asked to select one of three possible actions: A, B or $\mathbf{C}$. Once all the participants of your group have made their action choices, you will be randomly paired with another participant linked to you in the network (all three participants linked to you will be equally likely to be your counterpart for the round).

Both your payoff and the payoff of your counterpart for the round will be determined by the actions selected by both of you, as it is shown in the following matrix. In the matrix, the row indicates your selected action (A, B or C) and the column refers to the action selected by your pair (A, B or C). Thus each cell of the matrix shows your payoff (first number, in green) and the payoff of the other person (second number, in purple) associated to the combination of actions of yourself and of your pair indicated by the row and the column, respectively. 


\begin{tabular}{|c|c|c|c|c|}
\hline & \multicolumn{3}{|c|}{ Other person's choice } \\
\hline & & $\mathbf{A}$ & B & C \\
\hline \multirow{3}{*}{$\begin{array}{c}\text { Your } \\
\text { choice }\end{array}$} & $\mathbf{A}$ & $70, \quad 70$ & $80, \quad 0$ & $110, \quad 0$ \\
\hline & B & 0,80 & 90,90 & $110, \quad 0$ \\
\hline & C & 0,110 & 0,110 & 100,100 \\
\hline
\end{tabular}

This means that:

- If your choice is A and the choice of your pair is A, you earn $70 \mathrm{ECU}$ and your pair earns $70 \mathrm{ECU}$.

- If your choice is A and the choice of your pair is B, you earn $80 \mathrm{ECU}$ and your pair earns $0 \mathrm{ECU}$.

- If your choice is $\mathrm{A}$ and the choice of your pair is $\mathrm{C}$, you earn $110 \mathrm{ECU}$ and your pair earns 0 ECU.

- If your choice is B and the choice of your pair is A, you earn $\quad 0$ ECU and your pair earns 80 ECU.

- If your choice is B and the choice of your pair is B, you earn 90 ECU and your pair earns 90 ECU.

- If your choice is B and the choice of your pair is C, you earn 110 ECU and your pair earns 0 ECU.

- If your choice is $C$ and the choice of your pair is A, you earn $\quad 0$ ECU and your pair earns 110 ECU.

- If your choice is $C$ and the choice of your pair is B, you earn $\quad 0$ ECU and your pair earns 110 ECU.

- If your choice is $C$ and the choice of your pair is $C$, you earn 100 ECU and your pair earns 100 ECU.

6.- At the end of every round, you will get information about current and past rounds. The information consists of your position in the network, the position of your counterpart, your choice, the choice of your counterpart, and your (round) payoff.

7.- Payoffs. At the end of the experiment, you will be paid the earnings that you achieved in 4 rounds that will be randomly selected from the 40 rounds of play. One round will be chosen from the first 10 periods, one round will be chosen from the second 10 periods, one round will be chosen from the third 10 rounds, and one round will be selected from the last 10 periods. These earnings are transformed to cash at the exchange rate of $100 \mathrm{ECU}=6 €$. In addition, just by showing up, you will also be paid a fee of $€ 2.50$.

\section{Instructions treatment Closed_Clust}

The instructions of treatment Closed_Clust are analogous to those of treatment Open_Clust except for points 3 and 4 , which are replaced by the following:

3.- Each round consists of two stages: first, communication stage, and second, action stage. Your final earnings will depend only on what you choose and what others in your group choose in the 
action stage. Three actions, A, B and C, are available in the action stage. The communication stage that precedes the action stage involves each participant sending messages. Three messages are available in the communication stage, and they shall be labelled by the same letters, A, B and C, as the actions available in the action stage. A message may indicate your intended action in the subsequent action stage. However, you do not have to follow your message when it comes to making an action choice. We now describe each of these two stages in more detail.

4.- Communication stage. This stage consists of 90 seconds in which each participant of the group can send messages to those ones he/she is linked to in the network. During this stage, at each point in time, the participants send messages by marking an option: A, B or C. The option (A, B or C) marked by a participant will be immediately visible in the screens of all the participants linked to him/her in the network. Each participant can change the marked option at any time during this stage, and the new option will immediately be updated in the screens of the participants linked to him/her. When the time finishes, the participants can no longer send any new message.

For example, the participant in position 4 will observe the messages of the participants in positions 2, 3, 5 and 6 (and vice versa), but not those of participants in positions 1, 7 and 8.

\section{Instructions treatment NoComm_Clust}

The instructions of treatment NoComm_Clust are analogous to those of treatment Open_Clust except for points 3 and 4, which are removed. 


\section{Appendix B: Proofs}

\section{Proof of Proposition 1.}

The result is proved by induction arguments: We first prove that, if the result is true in time interval $t-1$, then it is true in interval $t$ (Lemma 1 ). Then, we prove that the result is true in interval 3 (Lemma 2).

Let $b(y ; N, p)$ and $B(y ; N, p)$ be, respectively, the density and the cumulative function of the binomial distribution of getting $y$ successes with parameters $N$ and $p$. Let $f_{t}^{g}(x ; p)$ and $F_{t}^{g}(x ; p)$ be, respectively, the density and the cumulative functions of the probability distribution on the number $x$ of subjects that have sent at least one message in interval $t$ or in one of the previous intervals in network $g \in\{c, n c\}$, given the diffusion probability $p$, where $c$ and $n c$ stand for networks Clust and NoClust, respectively.

Lemma 1: For each $p \in(0,1)$ and $t>3$, if $f_{t-1}^{n c}(x ; p)$ first order stochastically dominates $f_{t-1}^{c}(x ; p)$, then $f_{t}^{n c}(x ; p)$ first order stochastically dominates $f_{t}^{c}(x ; p)$.

Proof:

Consider network NoClust $(g=n c)$. In the event that no subject has sent a message until interval $t-1$ (included), only the four subjects that received a message from the seed player have a strictly positive probability to send a message. In the event that $i>0$ subjects have sent a message until interval $t-1$ (included), all other $7-i$ subjects have received at least one message and have a strictly positive probability to send a message. Given these considerations, we can write:

$$
f_{t}^{n c}(x ; p)=\sum_{i=0}^{7} f_{t-1}^{n c}(i ; p) \cdot w_{i}^{n c}(x ; p),
$$

where

$$
w_{i}^{n c}(x ; p)=\left\{\begin{array}{cl}
b(x ; 4, p) & \text { if } i=0 \\
b(x-i ; 7-i, p) & \text { if } 1 \leq i \leq 6 \\
0 & \text { if } i=7
\end{array}\right.
$$

Arranging $F_{t}^{n c}(x ; p)=\sum_{j=0}^{x} f_{t}^{n c}(j ; p)$, we can write: 


$$
F_{t}^{n c}(x ; p)=\sum_{i=0}^{7} f_{t-1}^{n c}(i ; p) \cdot W_{i}^{n c}(x ; p)
$$

where

$$
W_{i}^{n c}(x ; p)=\left\{\begin{array}{cl}
B(x ; 4, p) & \text { if } i=0 \\
B(x-i ; 7-i, p) & \text { if } 1 \leq i \leq 6 \\
0 & \text { if } i=7
\end{array}\right.
$$

Claim 1. $W_{i}^{n c}(x ; p)$ is weakly decreasing in i, i.e., for all $i \in\{1, \ldots, 6\}, W_{i}^{n c}(x ; p) \geq W_{i+1}^{n c}(x ; p)$. The proof of Claim 1 follows from a direct comparison of the binomial distributions included in function $W_{i}^{n c}(x ; p)$ (computations are available upon request).

Consider now network Clust $(g=c)$. In the event that no subject has sent a message until interval $t-1$ (included), only the four subjects that received a message from the seed player have a strictly positive probability to send a message. In the event that $i \geq 3$ subjects have sent a message until interval $t-1$ (included), all other $7-i$ subjects have received at least one message and have a strictly positive probability to send a message. In the event that $i \in\{1,2\}$ subjects have sent a message until interval $t-1$ (included), either 4 or 5 other subjects have received at least one message and have a strictly positive probability to send a message. Given these considerations, we can write:

$$
f_{t}^{c}(x ; p)=\sum_{i=0}^{7} f_{t-1}^{c}(i ; p) \cdot w_{i}^{c}(x ; p)
$$

where

$$
w_{i}^{c}(x ; p)=\left\{\begin{array}{cc}
b(x ; 4, p) & \text { if } i=0 \\
h_{i} \cdot b(x-i ; 5, p)+\left(1-h_{i}\right) b(x-i ; 4, p), h_{i} \in(0,1) & \text { if } i=1,2 \\
b(x-i ; 7-i, p) & \text { if } 3 \leq i \leq 6 \\
0 & \text { if } i=7
\end{array}\right.
$$

Arranging $F_{t}^{c}(x ; p)=\sum_{i=0}^{x} f_{t}^{c}(i ; p)$, we can write:

$$
F_{t}^{c}(x ; p)=\sum_{i=0}^{7} f_{t-1}^{c}(i ; p) \cdot W_{i}^{c}(x ; p)
$$

where

$$
W_{i}^{c}(x ; p)=\left\{\begin{array}{cc}
B(x ; 4, p) & \text { if } i=0 \\
h_{i} B(x-i ; 5, p)+\left(1-h_{i}\right) B(x-i ; 4, p), h_{i} \in(0,1) & \text { if } i=1,2 \\
B(x-i ; 7-i, p) & \text { if } 3 \leq i \leq 6 \\
0 \quad & \text { if } i=7
\end{array}\right.
$$


We now prove that $F_{t}^{n c}(x ; p) \leq F_{t}^{c}(x ; p), \forall x$, i.e.,

$$
\sum_{i=0}^{7} f_{t-1}^{n c}(i ; p) \cdot W_{i}^{n c}(x ; p) \leq \sum_{i=0}^{7} f_{t-1}^{c}(i ; p) \cdot W_{i}^{c}(x ; p), \forall x \text {. }
$$

Claim 2. $W_{i}^{c}(x ; p)>W_{i}^{n c}(x ; p), i=1,2$.

The proof of Claim 2 follows from a direct comparison of the binomial distributions included in the functions $W_{i}^{n c}(x ; p)$ and $W_{i}^{c}(x ; p)$, given in (4) and (8) (computations are available upon request). Moreover, by comparing (4) and (8), we can immediately state the following remark.

Remark 1. $W_{i}^{c}(x ; p)=W_{i}^{n c}(x ; p), i \neq 1,2$.

Given Claim 2 and Remark 1, to prove that (9) holds, it suffices to prove the following inequality:

$$
\sum_{i=0}^{7} f_{t-1}^{n c}(i ; p) \cdot W_{i}^{n c}(x ; p) \leq \sum_{i=0}^{7} f_{t-1}^{c}(i ; p) \cdot W_{i}^{n c}(x ; p), \forall x,
$$

where we have replaced $W_{i}^{c}(x ; p)$ by $W_{i}^{n c}(x ; p)$ in the RHS of (9). Given that $f_{t-1}^{n c}(x ; p)$ first order stochastically dominates $f_{t-1}^{c}(x ; p)$ and Claim 1 , standard results in expected utility theory on first order stochastic dominance prove inequality (10). This completes the proof of Lemma 1.

Lemma 2: For all $p \in(0,1), f_{3}^{n c}(x ; p)$ first order stochastically dominates $f_{3}^{c}(x ; p)$.

Proof:

Since in period 1 the seed player sends a message that is received by four subjects, these four subjects are the only ones with a positive probability to send a message in period 2 and, therefore, we can state the following remark.

Remark 2. For all $p \in(0,1), f_{2}^{n c}(x ; p)=f_{2}^{c}(x ; p)=b(x ; 4, p), \forall x$.

Consider network NoClust $(g=n c)$ in $t=3$. In the event that no subject sent a message in period 2 , only the four subjects that received a message from the seed player have a strictly positive probability to send a message. In the event that $i>0$ subjects sent a message in period 2 , all other $7-i$ subjects have received at least one message and, therefore, have a strictly positive probability to send a message. Given these considerations we can write:

$$
f_{3}^{n c}(x ; p)=\sum_{i=0}^{4} f_{2}^{n c}(i ; p) \cdot w_{i}^{n c}(x ; p),
$$

where $w_{i}^{n c}(x ; p)$ is defined in (2). Arranging $F_{3}^{n c}(x ; p)=\sum_{i=0}^{x} f_{3}^{n c}(i ; p)$, we can write: 


$$
F_{3}^{n c}(x ; p)=\sum_{i=0}^{4} f_{2}^{n c}(i ; p) \cdot W_{i}^{n c}(x ; p),
$$

where $W_{i}^{n c}(x ; p)$ is defined in (4).

Consider now network Clust $(g=c)$ in $t=3$. In the event that no subject sent a message in period 2 , only the four subjects that received a message from the seed player have a strictly positive probability to send a message. In the event that $i \geq 3$ subjects sent a message in period 2 , all other $7-i$ subjects have received at least one message and, therefore, have a strictly positive probability to send a message. In the event that $i \in\{1,2\}$ subjects sent a message in period 2 , either other 4 or 5 subjects have received at least one message, and have a strictly positive probability to send a message. Given these considerations, we can write:

$$
f_{3}^{c}(x ; p)=\sum_{i=0}^{4} f_{2}^{c}(i ; p) \cdot w_{i}^{c}(x ; p),
$$

where $w_{i}^{c}(x ; p)$ is defined in (6). Arranging $F_{3}^{c}(x ; p)=\sum_{i=0}^{x} f_{3}^{c}(i ; p)$, we can write:

$$
F_{3}^{c}(x ; p)=\sum_{i=0}^{4} f_{2}^{c}(i ; p) \cdot W_{i}^{c}(x ; p),
$$

where $W_{i}^{c}(x ; p)$ is defined in (8).

We need to prove that $F_{3}^{n c}(x ; p) \leq F_{3}^{c}(x ; p), \forall x$, i.e.,

$$
\sum_{i=0}^{4} f_{2}^{n c}(i ; p) \cdot W_{i}^{n c}(x ; p) \leq \sum_{i=0}^{4} f_{2}^{c}(i ; p) \cdot W_{i}^{c}(x ; p), \forall x
$$

By Claim 2 and Remarks 1 and 2, inequality (11) holds. This completes the proof of Lemma 2. By induction, results in Lemma 1 and Lemma 2 prove Proposition 1. QED

\section{Proof of Proposition 2.}

In Figure B1 we represent the normal form of the extended Stag Hunt game (cf. Figure 1), accounting for the efficiency bonus $b$ and the cost of lying $x^{i}, i \in\{$ Open,Closed $\}$, either with (i) or (ii) without player 1 sending a message (NoM vs. M). 
Figure B1. Extended Stag Hunt game accounting for efficiency and cost of lying

(i) NoM (No Message)

Player 2

\begin{tabular}{|c|c|c|c|c|}
\cline { 3 - 5 } \multicolumn{1}{c|}{} & \multicolumn{1}{c|}{$\boldsymbol{A}$} & $\boldsymbol{B}$ & $\boldsymbol{C}$ \\
\cline { 2 - 5 } Player 1 & $\boldsymbol{A}$ & 70,70 & 80,0 & 110,0 \\
\cline { 2 - 5 } & $\boldsymbol{B}$ & 0,80 & 90,90 & 110,0 \\
\cline { 2 - 5 } & $\boldsymbol{C}$ & 0,110 & 0,110 & $100+b, 100+b$ \\
\hline
\end{tabular}

(ii) M (Message)

Player 2

\begin{tabular}{|c|c|c|c|c|}
\cline { 2 - 5 } \multicolumn{1}{c|}{} & $\boldsymbol{A}$ & $\boldsymbol{B}$ & $\boldsymbol{C}$ \\
\hline \multirow{4}{*}{ Player 1 } & $\boldsymbol{A}$ & $70-x^{i}, 70$ & $80-x^{i}, 0$ & $110-x^{i}, 0$ \\
\cline { 2 - 5 } & $\boldsymbol{B}$ & $-x^{i}, 80$ & $90-x^{i}, 90$ & $110-x^{i}, 0$ \\
\cline { 2 - 5 } & $\boldsymbol{C}$ & 0,110 & 0,110 & $100+b, 100+b$ \\
\hline
\end{tabular}

In the whole game, the set of strategies of player 1 is

$$
S_{1}=\{(N o M, A),(N o M, B),(N o M, C),(M, A),(M, B),(M, C)\},
$$

where, for simplicity, we are considering that player 1 takes simultaneously the decision of whether to send a (C-)message or not (i.e., $M$ or $N o M)$ and the action to play $(A, B$, or $C) .{ }^{19}$ The set of strategies of player 2 is

$$
S_{2}=\{(A, A),(A, B),(A, C),(B, A),(B, B),(B, C),(C, A),(C, B),(C, C)\},
$$

where $(y, z)$ means to play action $y \in\{A, B, C\}$ after NoM and action $z \in\{A, B, C\}$ after $M$. In Figure B2 we present the normal form of the whole game, including only the payoffs of player 1.

Figure B2. Normal form of the (whole) bilateral game with player 1's payoffs

\begin{tabular}{|c|c|c|c|c|c|c|c|c|c|}
\cline { 2 - 10 } \multicolumn{1}{c|}{} & $\boldsymbol{A}, \boldsymbol{A}$ & $\boldsymbol{A}, \boldsymbol{B}$ & $\boldsymbol{A}, \boldsymbol{C}$ & $\boldsymbol{B}, \boldsymbol{A}$ & $\boldsymbol{B}, \boldsymbol{B}$ & $\boldsymbol{B}, \boldsymbol{C}$ & $\boldsymbol{C}, \boldsymbol{A}$ & $\boldsymbol{C}, \boldsymbol{B}$ & $\boldsymbol{C}, \boldsymbol{C}$ \\
\hline NoM, $\boldsymbol{A}$ & 70 & 70 & 70 & 80 & 80 & 80 & 110 & 110 & 110 \\
\hline NoM, $\boldsymbol{B}$ & 0 & 0 & 0 & 90 & 90 & 90 & 110 & 110 & 110 \\
\hline NoM, $\boldsymbol{C}$ & 0 & 0 & 0 & 0 & 0 & 0 & $100+b$ & $100+b$ & $100+b$ \\
\hline $\boldsymbol{M}, \boldsymbol{A}$ & $70-x^{i}$ & $80-x^{i}$ & $110-x^{i}$ & $70-x^{i}$ & $80-x^{i}$ & $110-x^{i}$ & $70-x^{i}$ & $80-x^{i}$ & $110-x^{i}$ \\
\hline $\boldsymbol{M}, \boldsymbol{B}$ & $-x^{i}$ & $90-x^{i}$ & $110-x^{i}$ & $-x^{i}$ & $90-x^{i}$ & $110-x^{i}$ & $-x^{i}$ & $90-x^{i}$ & $110-x^{i}$ \\
\hline $\boldsymbol{M}, \boldsymbol{C}$ & 0 & 0 & $100+b$ & 0 & 0 & $100+b$ & 0 & 0 & $100+b$ \\
\hline
\end{tabular}

Strategies $(M, A)$ and $(M, B)$ are dominated by $(N o M, A)$ if $x^{i}>40$, and strategy $(M, C)$ is not dominated. Therefore, if $x^{i}>40$, to play A and B after sending a (C-)message are dominated (by

\footnotetext{
${ }^{19}$ Note that the set of strategy in the extensive form of the game is larger. Given that player 1 sequentially takes the two decisions our simplification is innocuous.
} 
not sending a message and playing A). Hence, if player 2 observes a (C-)message, he should infer that player 1 intends to play C.

If the bonus $b$ is higher than 10 , then after receiving a (C-)message player 2's best response is to play C (see right panel in Figure B1). Hence the message is credible if $x^{i}>\bar{x}=40$ and $b>10$. The second part of the proposition follows from considering all pairs $\left(x^{\text {open }}, x^{\text {closed }}\right)$ such that $x^{\text {open }}>40>x^{\text {closed }}$. QED 


\section{Appendix C: Statistical and econometric analysis}

Table C1. Likelihood (\%) of actions A, B and C, by treatment and blocks of $\mathbf{1 0}$ periods

\begin{tabular}{|c|c|c|c|c|c|c|c|c|}
\hline & \multicolumn{4}{|c|}{ NoComm_Clust } & \multicolumn{4}{|c|}{ NoComm_NoClust } \\
\hline & $1-10$ & $11-20$ & $21-30$ & $31-40$ & $1-10$ & $11-20$ & $21-30$ & $31-40$ \\
\hline A & $\begin{array}{l}70.31 \\
(450)\end{array}$ & $\begin{array}{r}94.38 \\
(604)\end{array}$ & $\begin{array}{r}96.25 \\
(616)\end{array}$ & $\begin{array}{r}95.47 \\
(611)\end{array}$ & $\begin{array}{l}59.69 \\
(382)\end{array}$ & $\begin{array}{r}90.78 \\
(581)\end{array}$ & $\begin{array}{r}95.47 \\
(611)\end{array}$ & $\begin{array}{r}98.59 \\
(631)\end{array}$ \\
\hline B & $\begin{array}{l}5.16 \\
(33)\end{array}$ & $\begin{array}{l}2.03 \\
(13)\end{array}$ & $\begin{array}{r}0.94 \\
(6)\end{array}$ & $\begin{array}{r}0.78 \\
(5)\end{array}$ & $\begin{array}{r}11.72 \\
(75)\end{array}$ & $\begin{array}{l}3.44 \\
(22)\end{array}$ & $\begin{array}{l}1.88 \\
(12)\end{array}$ & $\begin{array}{r}0.63 \\
(4)\end{array}$ \\
\hline C & $\begin{array}{r}24.53 \\
(157)\end{array}$ & $\begin{array}{l}3.59 \\
(23)\end{array}$ & $\begin{array}{l}2.81 \\
(18)\end{array}$ & $\begin{array}{l}3.75 \\
(24)\end{array}$ & $\begin{array}{r}28.59 \\
(183)\end{array}$ & $\begin{array}{l}5.78 \\
(37)\end{array}$ & $\begin{array}{l}2.66 \\
(17)\end{array}$ & $\begin{array}{r}0.78 \\
(5)\end{array}$ \\
\hline Total & $\begin{array}{r}100 \\
(640)\end{array}$ & $\begin{array}{r}100 \\
(640)\end{array}$ & $\begin{array}{r}100 \\
(640)\end{array}$ & $\begin{array}{r}100 \\
(640)\end{array}$ & $\begin{array}{r}100 \\
(640)\end{array}$ & $\begin{array}{r}100 \\
(640)\end{array}$ & $\begin{array}{r}100 \\
(640)\end{array}$ & $\begin{array}{r}100 \\
(640)\end{array}$ \\
\hline & \multicolumn{4}{|c|}{ Closed_Clust } & \multicolumn{4}{|c|}{ Closed_NoClust } \\
\hline & $1-10$ & $11-20$ & $21-30$ & $31-40$ & $1-10$ & $11-20$ & $21-30$ & $31-40$ \\
\hline A & $\begin{array}{l}51.67 \\
(248)\end{array}$ & $\begin{array}{l}90.63 \\
(435)\end{array}$ & $\begin{array}{r}97.29 \\
(467)\end{array}$ & $\begin{array}{l}98.96 \\
(475)\end{array}$ & $\begin{array}{l}47.66 \\
(305)\end{array}$ & $\begin{array}{l}87.03 \\
(557)\end{array}$ & $\begin{array}{r}92.34 \\
(591)\end{array}$ & $\begin{array}{r}95.16 \\
(609)\end{array}$ \\
\hline B & $\begin{array}{l}6.46 \\
(31)\end{array}$ & $\begin{array}{r}1.46 \\
(7)\end{array}$ & $\begin{array}{r}0.83 \\
(4)\end{array}$ & $\begin{array}{r}0 \\
(0)\end{array}$ & $\begin{array}{l}7.34 \\
(47)\end{array}$ & $\begin{array}{l}3.75 \\
(24)\end{array}$ & $\begin{array}{l}2.81 \\
(18)\end{array}$ & $\begin{array}{l}3.13 \\
(20)\end{array}$ \\
\hline C & $\begin{array}{l}41.88 \\
(201)\end{array}$ & $\begin{array}{l}7.92 \\
(38)\end{array}$ & $\begin{array}{r}1.88 \\
(9)\end{array}$ & $\begin{array}{r}1.04 \\
(5)\end{array}$ & $\begin{array}{r}45 \\
(288)\end{array}$ & $\begin{array}{l}9.22 \\
(59)\end{array}$ & $\begin{array}{l}4.84 \\
(31)\end{array}$ & $\begin{array}{l}1.72 \\
(11)\end{array}$ \\
\hline Total & $\begin{array}{r}100 \\
(480)\end{array}$ & $\begin{array}{r}100 \\
(480)\end{array}$ & $\begin{array}{r}100 \\
(480)\end{array}$ & $\begin{array}{r}100 \\
(480)\end{array}$ & $\begin{array}{r}100 \\
(640)\end{array}$ & $\begin{array}{r}100 \\
(640)\end{array}$ & $\begin{array}{r}100 \\
(640)\end{array}$ & $\begin{array}{r}100 \\
(640)\end{array}$ \\
\hline & \multicolumn{4}{|c|}{ Open_Clust } & \multicolumn{4}{|c|}{ Open_NoClust } \\
\hline & $1-10$ & $11-20$ & $21-30$ & $31-40$ & $1-10$ & $11-20$ & $21-30$ & $31-40$ \\
\hline A & $\begin{array}{l}5.63 \\
(36)\end{array}$ & $\begin{array}{l}6.09 \\
(39)\end{array}$ & $\begin{array}{l}5.63 \\
(36)\end{array}$ & $\begin{array}{l}9.38 \\
(60)\end{array}$ & $\begin{array}{r}0.47 \\
(3)\end{array}$ & $\begin{array}{r}0 \\
(0)\end{array}$ & $\begin{array}{r}0.16 \\
(1)\end{array}$ & $\begin{array}{l}2.81 \\
(18)\end{array}$ \\
\hline B & $\begin{array}{l}1.72 \\
(11)\end{array}$ & $\begin{array}{r}0.63 \\
(4)\end{array}$ & $\begin{array}{r}0.31 \\
(2)\end{array}$ & $\begin{array}{r}0.63 \\
(4)\end{array}$ & $\begin{array}{r}0 \\
(0)\end{array}$ & $\begin{array}{r}0 \\
(0)\end{array}$ & $\begin{array}{r}0 \\
(0)\end{array}$ & $\begin{array}{r}0.31 \\
(2)\end{array}$ \\
\hline C & $\begin{array}{r}92.66 \\
(593)\end{array}$ & $\begin{array}{r}93.28 \\
(597)\end{array}$ & $\begin{array}{l}94.06 \\
(602)\end{array}$ & $\begin{array}{r}90 \\
(576)\end{array}$ & $\begin{array}{r}99.53 \\
(637)\end{array}$ & $\begin{array}{r}100 \\
(640)\end{array}$ & $\begin{array}{r}99.84 \\
(639)\end{array}$ & $\begin{array}{r}96.88 \\
(620)\end{array}$ \\
\hline Total & $\begin{array}{r}100 \\
(640)\end{array}$ & $\begin{array}{r}100 \\
(640)\end{array}$ & $\begin{array}{r}100 \\
640)\end{array}$ & $\begin{array}{r}100 \\
(640)\end{array}$ & $\begin{array}{r}100 \\
(640)\end{array}$ & $\begin{array}{r}100 \\
(640)\end{array}$ & $\begin{array}{r}100 \\
(640)\end{array}$ & $\begin{array}{r}100 \\
(640)\end{array}$ \\
\hline
\end{tabular}

The bottom number in each cell (in parentheses) is the number of observations. 
Table C2. Frequencies of choices by matching groups

\begin{tabular}{|c|c|c|c|}
\hline \multirow[t]{2}{*}{ Treatment - Matching Group } & \multicolumn{3}{|c|}{ Action } \\
\hline & A & B & C \\
\hline Nocomm_Clust - 1 & 94.38 & 0.47 & 5.16 \\
\hline Nocomm_Clust - 2 & 92.34 & 3.28 & 4.38 \\
\hline Nocomm_Clust - 3 & 80.78 & 4.06 & 15.16 \\
\hline Nocomm_Clust - 4 & 88.91 & 1.09 & 10.00 \\
\hline Nocomm_NoClust - 1 & 87.50 & 9.06 & 3.44 \\
\hline Nocomm_NoClust - 2 & 94.69 & 1.25 & 4.06 \\
\hline Nocomm_NoClust-3 & 73.13 & 4.06 & 22.81 \\
\hline Nocomm_NoClust-4 & 89.22 & 3.28 & 7.50 \\
\hline Closed_Clust - 1 & 88.59 & 1.72 & 9.69 \\
\hline Closed_Clust - 2 & 86.09 & 1.56 & 12.34 \\
\hline Closed_Clust - 3 & 79.22 & 3.28 & 17.50 \\
\hline Closed_NoClust - 1 & 89.38 & 1.25 & 9.38 \\
\hline Closed_NoClust - 2 & 72.34 & 9.22 & 18.44 \\
\hline Closed_NoClust - 3 & 87.66 & 1.56 & 10.78 \\
\hline Closed_NoClust - 4 & 72.81 & 5.00 & 22.19 \\
\hline Open_Clust - 1 & 23.59 & 0.16 & 76.25 \\
\hline Open_Clust - 2 & 2.81 & 0.00 & 97.19 \\
\hline Open_Clust - 3 & 0.16 & 0.94 & 98.91 \\
\hline Open_Clust - 4 & 0.16 & 2.19 & 97.66 \\
\hline Open_NoClust - 1 & 0.31 & 0.16 & 99.53 \\
\hline Open_NoClust - 2 & 0.16 & 0.00 & 99.84 \\
\hline Open_NoClust - 3 & 0.16 & 0.00 & 99.84 \\
\hline Open_NoClust - 4 & 2.81 & 0.16 & 97.03 \\
\hline
\end{tabular}


Figure C1. Evolution of frequencies of A, B and C-choices by matching groups

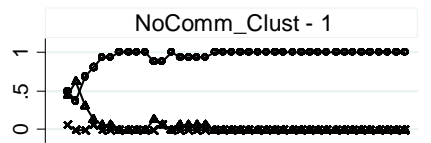

NoComm_NoClust - 1

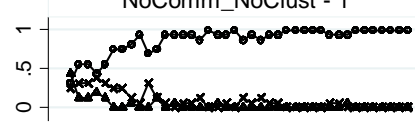

Closed_Clust - 1

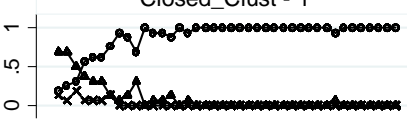

Closed_NoClust - 1

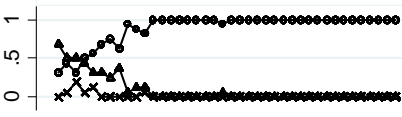

Open_Clust - 1
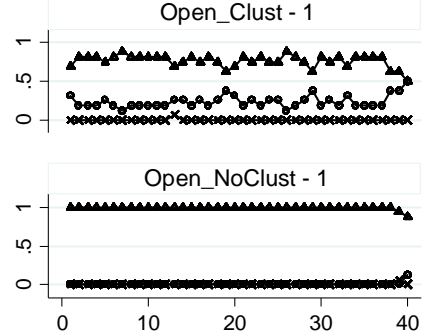

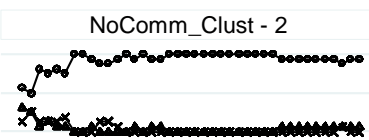

NoComm_NoClust - 2

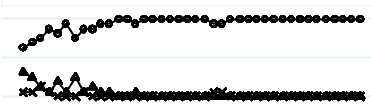

Closed_Clust - 2

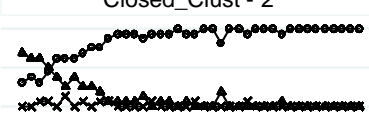

Closed_NoClust - 2

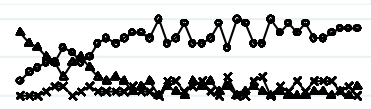

Open_Clust - 2

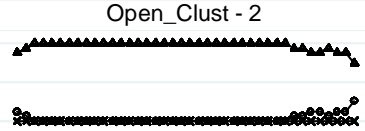

Open_NoClust - 2

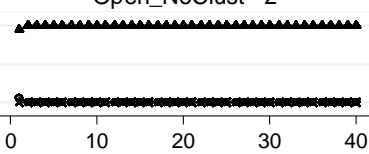

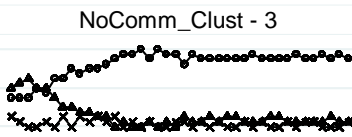

NoComm_NoClust - 3

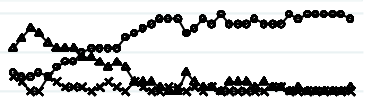

Closed_Clust - 3

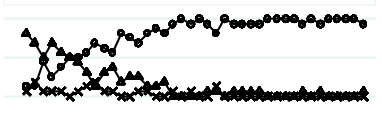

Closed_NoClust - 3

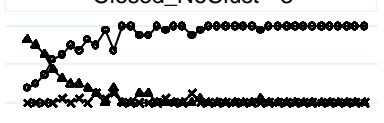

Open_Clust - 3

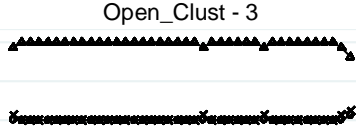

Open_NoClust - 3

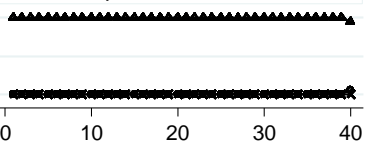

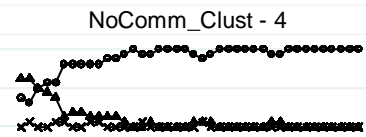

NoComm_NoClust - 4

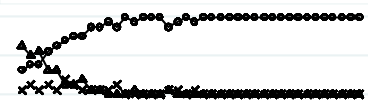

Closed_NoClust - 4

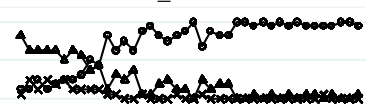

Open Clust - 4
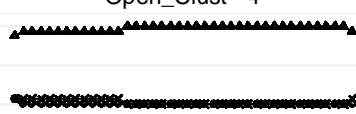

Open_NoClust - 4

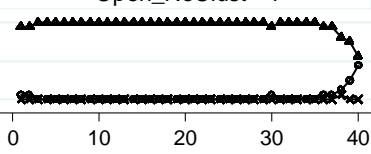

Period

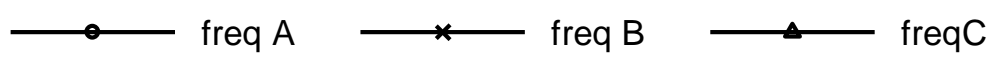


Table C3. Logit models on the choice of action C

\begin{tabular}{|c|c|c|c|c|c|c|c|}
\hline & (1) & (2) & (3) & (4) & (5) & (6) & (7) \\
\hline VARIABLES & No Comm & $\begin{array}{l}\text { Closed } \\
\text { Comm }\end{array}$ & $\begin{array}{l}\text { Open } \\
\text { Comm }\end{array}$ & $\begin{array}{l}\text { Closed } \\
\text { Comm }\end{array}$ & $\begin{array}{l}\text { Open } \\
\text { Comm }\end{array}$ & $\begin{array}{l}\text { Closed } \\
\text { Comm }\end{array}$ & $\begin{array}{l}\text { Open } \\
\text { Comm }\end{array}$ \\
\hline $\begin{array}{l}\text { Period } \\
\text { at Net_NoClust }=0\end{array}$ & $\begin{array}{c}-0.114^{* * *} \\
(0.032)\end{array}$ & $\begin{array}{c}-0.200 * * * \\
(0.023)\end{array}$ & $\begin{array}{l}-0.014 \\
(0.014)\end{array}$ & $\begin{array}{c}-0.097 * * * \\
(0.018)\end{array}$ & $\begin{array}{l}-0.008 \\
(0.015)\end{array}$ & $\begin{array}{l}-0.035 \\
(0.032)\end{array}$ & $\begin{array}{l}-0.007 \\
(0.014)\end{array}$ \\
\hline Period & $-0.180 * * *$ & $-0.164 * * *$ & $-0.158 * * *$ & $-0.110^{* * *}$ & $-0.151^{* * *}$ & $-0.047 * * *$ & $-0.143^{* * *}$ \\
\hline at Net_NoClust $=1$ & $(0.035)$ & $(0.023)$ & $(0.047)$ & $(0.026)$ & $(0.047)$ & $(0.014)$ & $(0.043)$ \\
\hline Net_NoClust & $\begin{array}{c}0.228 \\
(0.435)\end{array}$ & $\begin{array}{l}0.0637 \\
(0.419)\end{array}$ & $\begin{array}{l}3.950 * * \\
(1.622)\end{array}$ & $\begin{array}{c}0.272 \\
(0.389)\end{array}$ & $\begin{array}{l}3.815^{* *} \\
(1.621)\end{array}$ & $\begin{array}{c}0.170 \\
(0.269)\end{array}$ & $\begin{array}{l}3.597 * * \\
(1.504)\end{array}$ \\
\hline Mess_C_rec & & & & $\begin{array}{c}0.096 \\
(0.257)\end{array}$ & $\begin{array}{c}0.066 \\
(0.268)\end{array}$ & $\begin{array}{c}0.102 \\
(0.282)\end{array}$ & $\begin{array}{c}0.128 \\
(0.275)\end{array}$ \\
\hline Mess_C_sent & & & & $\begin{array}{c}0.420 \\
(0.590)\end{array}$ & $\begin{array}{c}0.099 \\
(0.709)\end{array}$ & $\begin{array}{c}0.384 \\
(0.586)\end{array}$ & $\begin{array}{c}0.100 \\
(0.661)\end{array}$ \\
\hline $\begin{array}{l}\text { Mess_C_rec } \\
\text { \# Mess_C_sent }\end{array}$ & & & & $\begin{array}{c}0.769 * * * \\
(0.240)\end{array}$ & $\begin{array}{c}0.317 * * * \\
(0.107)\end{array}$ & $\begin{array}{c}0.734^{* * *} \\
(0.249)\end{array}$ & $\begin{array}{c}0.295^{* * *} \\
(0.113)\end{array}$ \\
\hline $\begin{array}{l}\text { Credibility } \\
(\gamma=0.7)\end{array}$ & & & & & & $\begin{array}{c}2.506^{* * *} \\
(0.818)\end{array}$ & \\
\hline $\begin{array}{l}\text { Credibility } \\
(\gamma=0.4)\end{array}$ & & & & & & & $\begin{array}{c}2.286 * * * \\
(0.571)\end{array}$ \\
\hline Constant & $\begin{array}{l}-0.473 \\
(0.344)\end{array}$ & $\begin{array}{c}1.217^{* * *} \\
(0.221)\end{array}$ & $\begin{array}{c}4.034^{* * *} \\
(0.312)\end{array}$ & $\begin{array}{c}-2.708^{* * *} \\
(0.769)\end{array}$ & $\begin{array}{c}3.407 * * * \\
(0.727)\end{array}$ & $\begin{array}{c}-4.595^{* * *} \\
(1.289)\end{array}$ & $\begin{array}{l}1.064^{*} \\
(0.614)\end{array}$ \\
\hline Observations & 5,120 & 4,480 & 5,120 & 4,480 & 5,120 & 4,384 & 5,110 \\
\hline
\end{tabular}

Robust standard errors in parentheses; ${ }^{* * *} p<0.01,{ }^{* *} p<0.05,{ }^{*} p<0.10$. 
Table C4. Stag Hunt game - Frequency of B-play by (treatment and) matching group

Matching (16-person) group

\begin{tabular}{|c|c|c|c|c|c|c|c|c|}
\cline { 2 - 8 } \multicolumn{1}{c|}{ Treatment } & 1 & 2 & 3 & 4 & 5 & 6 & 7 & 8 \\
\hline $\begin{array}{c}\text { No Comm } \\
\text { Network Clust }\end{array}$ & 0.0813 & 0.0891 & 0.1281 & 0.1594 & & & & \\
\hline $\begin{array}{c}\text { No Comm } \\
\text { Network NoClust }\end{array}$ & 0.0828 & 0.0906 & 0.1281 & 0.2188 & & & & \\
\hline $\begin{array}{c}\text { Closed Comm } \\
\text { Network Clust }\end{array}$ & 0.2609 & 0.3453 & 0.8719 & 0.9594 & 0.9672 & 0.9937 & 1.000 & 0.9969 \\
\hline $\begin{array}{c}\text { Closed Comm } \\
\text { Network NoClust }\end{array}$ & 0.2141 & 0.4953 & 0.5296 & 0.9187 & 0.9203 & 0.9391 & 0.9953 & 0.9 \\
\hline
\end{tabular}



Figure C2. Standard Stag Hunt - Average relative frequency of action B across
periods, by matching group

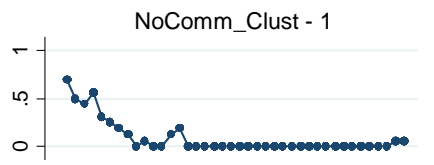

NoComm_NoClust - 1
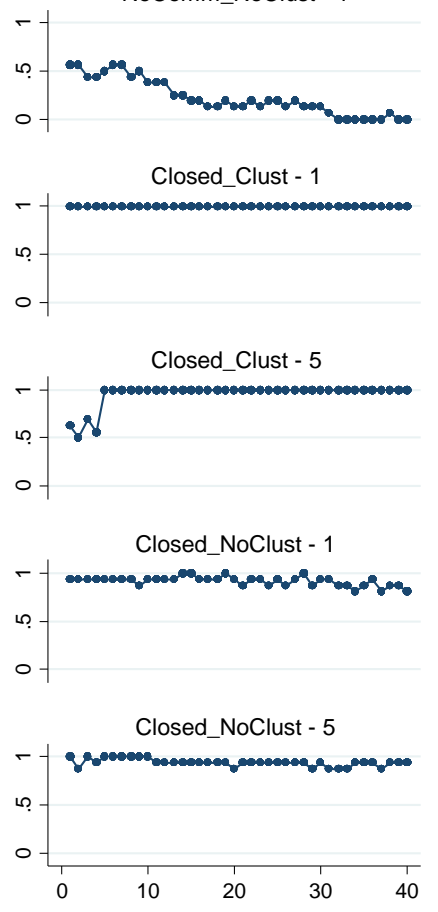

NoComm_Clust - 2

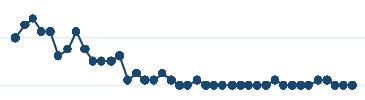

NoComm_NoClust - 2
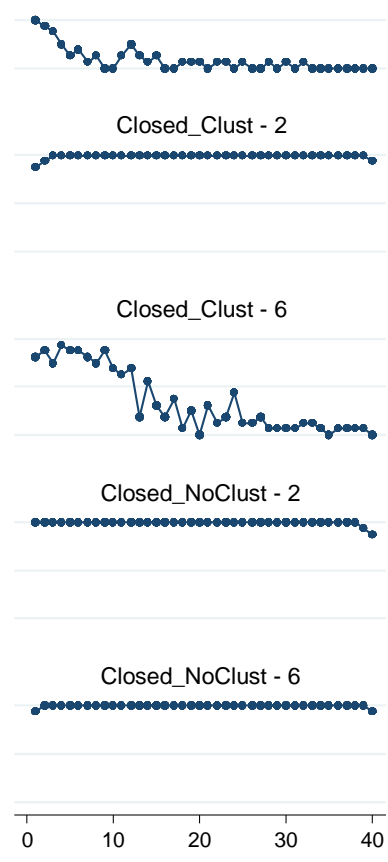

NoComm_Clust - 3

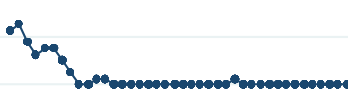

NoComm_NoClust - 3

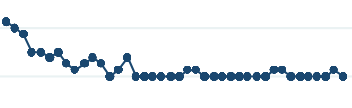

Closed_Clust - 3

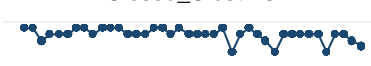

Closed_Clust - 7

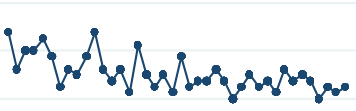

Closed_NoClust - 3

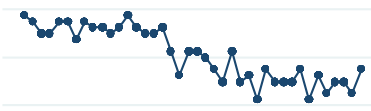

Closed_NoClust - 7

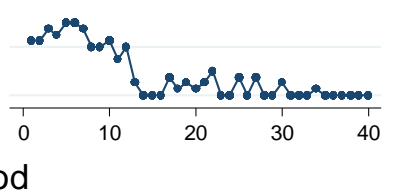

NoComm_Clust - 4

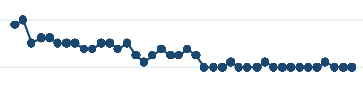

NoComm_NoClust - 4

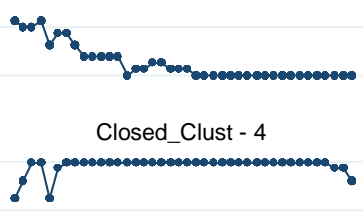

Closed_NoClust - 4
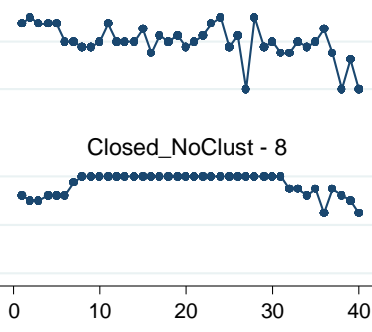

With abuse of notation (because here participants play a standard Stag Hunt game, not the extended one), in the Figure $\mathrm{C} 2$ we denote treatments with the following names:

- No communication, clustered network is named NoComm_Clust

- No communication, non-clustered network is named NoComm_NoClust

- Closed communication, clustered network is named Closed_Clust

- Closed communication, non-clustered network is named Closed_NoClust

The number after the name denotes the matching group. 